\title{
Diversité Floristique Des Ligneux Et Structure Des Formations À Garcinia Kola Heckel Dans Les Régions Du Centre Et De l'Est, Cameroun
}

\author{
Kamga Yanick Borel \\ Nguetsop Victor François \\ Momo Solefack Marie Caroline
}

Faculté des Sciences, Département de Biologie Végétale, Université de Dschang, Cameroun Unité de Recherche de Botanique Appliquée (URBOA) BP: 67, Dschang, Cameroun

\section{Riera Bernard}

Muséum National d'Histoire Naturelle. 4 avenue du Petit Château. F-91800 Brunoy, France

Doi: 10.19044/esj.2018.v14n21p451 URL:http://dx.doi.org/10.19044/esj.2018.v14n21p451

Abstract

In order to find the strategies to ensure the sustainable management of natural's resources and especially woody plants in the Congo Basin, a study was carried out on the floristic diversity of woody plants and the structure of Garcinia kola formations in the Centre and East regions of Cameroon. A total of 39 quadrats of $40 \mathrm{~m}$ x $40 \mathrm{~m}$ were installed in the Garcinia kola formations in these two regions. In each plot, we identified and counted all individuals with a dbh $\geq 10 \mathrm{~cm}$. Seedlings of $\mathrm{G}$. kola $(\mathrm{dbh}<10 \mathrm{~cm})$ were also counted in all the plots. The height and diameter of each individual were measured. ANOVA was used to compare the density averages of the different plots, and the DUNCAN test at 5\% significance level (XLSTAT 2017) was used to separate these mean values. This study confirms that the Clusiaceae (G. kola) forms the floristic background of the stand studied. The surveys revealed a total of 196 species, 156 species $\left(H^{\prime}=2.98\right)$ and 68 species $\left(H^{\prime}=2.13\right)$, respectively, identified in the East and Centre among the population of individuals showing $\mathrm{dbh} \geq 10 \mathrm{~cm}$. They were distributed into 124 genera and 46 families. The most diversified families in $G$. kola formations are Euphorbiaceae, Fabaceae, Meliaceae, Sterculiaceae, Moraceae, Anacardiaceae, Apocynaceae, Annonaceae, Rutaceae, Rubiaceae, and Clusiaceae. The average standing density of $G$. kola varies from 6.51 (East Region) to 40.72 (Centre Region) stems per hectare. The low density observed in the localities of the eastern region can be explained by the pressure exerted 
on this species in the natural environment linked mostly to methods of exploitation.

Keywords: Garcinia kola, NTFP, Diversity, Sustainable management, Centre- Est, Cameroon

\section{Résumé}

Afin de trouver des strategies pour une gestion durable des ressources naturelles et pariculièrement des plantes ligneuses dans le Bassin du Congo, une étude a été menée sur la diversité floristique des ligneux et la structure des formations à Garcinia kola dans les régions du Centre et 1'Est Cameroun. Au total 39 relevés de 40 m x 40 m ont été installés dans les formations à Garcinia kola dans ces deux régions. Dans chaque relevé les espèces présentes ont été identifiées, puis les individus ayant un diamètre à hauteur de poitrine (dhp) $\geq$ $10 \mathrm{~cm}$ ont été comptés. Les plantules des Garcinia kola $(\mathrm{dhp} \leq 10 \mathrm{~cm})$ ont également été comptées. L'ANOVA a été utilisée pour comparer les moyennes de densité des différents relevés et le test de DUNCAN au seuil de signification 5\% (XLSTAT 2017) pour séparer ces moyennes. Il ressort de cette étude que les Clusiaceae (G. kola) forment le fond floristique des peuplements étudiés. Les relevés comprennent au total 196 espèces, soit respectivement 156 espèces $\left(H^{\prime}=2,98\right)$ et 68 espèces $\left(H^{\prime}=2,13\right)$ recensées dans le peuplement montrant des individus à $\mathrm{dhp} \geq 10 \mathrm{~cm}$ à l'Est et au Centre ; elles se répartissent dans 124 genres et 46 familles.s. Les Euphorbiaceae, les Fabaceae, les Meliaceae, les Sterculiaceae, les Moraceae, les Anacardiaceae, les Apocynaceae, les Annonaceae, les Rutaceae, les Rubiaceae et les Clusiaceae sont les familles les plus diversifiées dans ces formations à G. kola . La densité moyenne sur pied de G. kola varie de 6,51 (Région de l'Est) à 40,72 (région du Centre) tiges par hectare. La faible densité observée dans la région de l'Est peut s'expliquer par la pression exercée sur cette espèce dans son milieu naturel lié probablement aux méthodes d'exploitation.

Mots-clés : Garcinia kola, Gestion durable, PFNL, Diversité, Régions Centre - Est, Cameroon

\section{Introduction}

Les forêts tropicales humides Africaines (FTHA) sont des écosystèmes terrestres recélant l'une des diversités biologiques les plus élevées et les plus complexes de la planète (Puig, 2001 ; Gemerden, 2004). Elles constituent le second massif forestier tropical humide, après celui du bassin amazonien. La richesse spécifique, tout comme la composition des communautés peut cependant varier significativement d'une région à l'autre. Une forte diversité floristique est par exemple observée sur les façades atlantiques couvrant 
l'Afrique centrale (l'Ouest du Cameroun et du Gabon) au centre du Kivu (République démocratique du Congo (RDC) et l'Afrique de l'Ouest (Sud de la Guinée, du Liberia et de la Côte d'ivoire). Par ailleurs ces zones de grandes richesses correspondent aux zones de forte pluviométrie et de faible saisonnalité, de températures moyennes faibles $\left(26^{\circ} \mathrm{C}\right)$ et montrent d'importantes variations altitudinales (Linder, 2001; Plana, 2004).

Les forêts guinéo-congolaises comprennent trois entités biogéographiques dont deux en Afrique centrale : les forêts du domaine bas guinéen qui s'étendent du Nigeria à la frontière orientale du Gabon; les forêts du domaine congolais localisées en République Démocratique du Congo (RDC) et au Congo Brazzaville; puis une entité en Afrique de l'Ouest, notamment les forêts du domaine haut-guinéen allant de la frontière Est du Ghana à 1'Ouest de la Guinée. Ces deux grands blocs sont séparés par une bande de savane (le 'Dahomey gap') situé entre le Togo et le Bénin. Malgré le relief, les forêts guinéo-congolaises se découpent ainsi en une mosaïque fine de formations végétales (forêt dense, des savanes, etc....), dominées par des arbres (White, 1986). Ces forêts sont sujettes aujourd'hui à une forte pression anthropique qui se superpose aux impacts climatiques à plus ou moins longues échelles de temps. En effet, de manière générale et principalement pour celles $\mathrm{du}$ bassin du Congo, elles sont soumises à une exploitation industrielle et constituent une source de revenus importante pour les populations forestières qui exploitent divers produits alimentaires, médicinaux etc. Au Cameroun par exemple, le secteur forestier contribue à hauteur de $10 \%$ du Produit Intérieur Brut (PIB) et environ 30\% des exportations (De Wasseige et al., 2012). Compte tenu de la richesse de ces forêts, celles-ci sont donc aujourd'hui menacées par l'exploitation forestière. De plus, la découverte et l'exploitation de nouveaux gisements miniers (or, uranium, diamant, cobalt etc..), couplée à de mauvaises pratiques agricoles notamment l'agriculture itinérante sur brûlis (manioc, haricot...), des cultures pérennes (palmier à huile, hévea etc...) et les changements globaux favorisent davantage la détérioration de ces écosystèmes forestiers. Ces exploitations demeurent donc une préoccupation écologique majeure, car elles menacent ainsi les services écosystémiques et pourraient contribuer à la disparition des espèces non encore identifiées ou en danger (Laurance, 2006).

Parmi les services écosystémiques rendus par espèces ligneuses de ces forêts, l'on peut insister sur les Produits Forestiers Non Ligneux (PFNL) qui sont d'un grand intérêt pour populations riveraines (Loubelo, 2012). Une gestion durable de ces formations forestières contribuerait directement à assurer une production à long terme des PFNL. Pourtant, depuis des décennies, les Produits Forestiers Non Ligneux (PFNL) ont été toujours relégués au second plan, l'exploitation du bois d'œuvre étant considérée comme la seule source des revenus issus de la forêt. Selon Eba'a et al. (2008), les PFNL 
constituent pourtant une partie non négligeable dans l'alimentation des populations vivant à proximité ou à l'intérieur des forêts tropicales africaines. Les pratiques d'exploitation sont variées et conduisent généralement à une mortalité des arbres et la rareté des ressources. Parmi les essences exploitées on peut citer Prunus africana, très répandu sur les habitats montagnards africains, elle est exploitée pour ses vertus curatives de divers maux dont l'adénome prostatique, Gnidia glauca, Irvingia spp., Cola acuminata, Gnetum africanum et Garcinia spp, dont G. kola. Des graines de Garcinia kola sont en effet l'un des nombreux produits forestiers non ligneux qui sont d'une grande importance socio-économique. Leur commercialisation sur les marchés nationaux et inter-nationaux améliore substantiellement le niveau de vie de ceux qui sont impliqués dans sa chaine de commercialisation dans les centres urbains et ruraux de la sous région (Adebisi, 2004). L'intérêt, sans cesse grandissant, porté à cette espèce dans le bassin du Congo et principalement au Cameroun pour son importance médicinale, économique et surtout les méthodes d'exploitation (abattage, annélation...) de l'écorce sont tels qu'il est souhaitable aujourd'hui de sensibiliser la population sur les techniques de récolte, de déterminer le potentiel sur pied des espèces à produit forestier non ligneux, les caractéristiques structurales des formations à $G$. kola afin de trouver des stratégies de gestion durable de la ressource. En effet, en Afrique tropicale et principalement au Cameroun, très peu de travaux ont été menés sur la caractérisation des peuplements de ligneux dont certains qui constituent le fond floristique sont producteurs de PFNL. On pourra ensuite s'intéresser à l'impact de leur exploitation sur la dynamique des populations de ces espèces.

Le présent travail qui a pour principal objectif de déterminer la diversité floristique et de caractériser les peuplements à base de G. kola dans deux régions du Cameroun, devra in fine contribuer à la valorisation des espèces à produits forestiers non ligneux en proposant des stratégies de gestion durable.

\section{Matériel et Méthodes \\ Sites de l'étude \\ Région du Centre}

La zone de Ndikinimiki - Makénénéqui a fait l'objet de cette étude. Elle est située dans la région du Centre (Cameroun), elle couvre la totalité du territoire des arrondissements de Ndikinimeki et de Makénéné, département du Mbam et Inoubou (Figure 1). Elle s'étend de $4^{\circ} 28^{\prime}$ à $5^{\circ} 00^{\prime}$ de latitude Nord et de $10^{\circ} 28^{\prime}$ à $11^{\circ} 00^{\prime}$ de longitude Est. Le relief de la zone est constitué de deux ensembles de plateaux de hautes et basses altitudes. D'après Bohin (1992), les sols de cette région sont de types ferralitiques rouges et jaunes. Les sols jaunes se retrouvent dans les basses terres de l'Ouest où les précipitations sont supérieures à $1700 \mathrm{~mm} / \mathrm{an}$. Le climat de la zone est du type équatorial à 
deux saisons des pluies entrecoupées de deux saisons sèches, la température moyenne enregistrée se situe au tour de $25^{\circ} \mathrm{C}$. La première et petite saison des pluies va de mars à juin, la seconde et la plus importante d'août à novembre. La grande saison sèche va de novembre à février et la petite de juin à août. Letouzey (1985) distingue dans cette zone six types de végétation. La forêt atlantique à Caesalpiniaceae rares, qui se caractérise par la rareté en Caesalpiniaceae. Mais aussi par la présence des éléments colonisateurs de forêts semi-caducifoliées s'installant ponctuellement à la faveur des chablis ou de la dégradation liée aux activités anthropiques. La forêt atlantique à Caesalpiniaceae rares, de type nord-occidental, sur relief bullé d'érosion, avec avancée d'éléments de forêts semi-caducifoliées. La forêt semi-caducifoliée à Sterculiaceae et Ulmaceae localisée au Nord de Ndikinimeki et à l'Est de Makénéné, se caractérise par une abondance des Sterculiaceae notamment les Cola spp et des Ulmaceae (Celtis spp). Les forêts semi-caducifoliées sur relief bullé d'érosion avec reliques d'éléments de forêts atlantiques à Caesalpiniaceae rares de type nord-occidental. Enfin des recrus de forêts semicaducifoliées, avec ou sans vestiges concentrés de savanes périforestières arbustives à Terminalia glaucescens sur relief bullé d'érosion, et avec avancée d'éléments soudano-zambéziens.

\section{Région de l'Est}

L'échantillonnage a été effectué dans plusieurs localités de cette région (Lomié, Aschi, Ngoïla, Dounzoh et Mpane Kobera) (Figure 1). La région connait un climat de type subtropical avec 4 saisons, deux saisons de pluies et deux saisons sèches. Les températures oscillent entre $18^{\circ} \mathrm{C}$ et $30^{\circ} \mathrm{C}$ (INS, 2010). Avec les précipitations moyennes de 1500-2000 mm/an, sauf dans les parties extrêmes de l'Est et du Nord, où elles sont légèrement plus faibles, cette zone est caractérisée par une humidité et une couverture nuageuse relativement élevées. Sur le plan géologique, la région est composée de formations précambriennes de la série du Dja inférieur ; les formations géologiques rencontrées sont les schistes et les grès quartzites du Bek. Ces formations sont observées sur l'axe Moloundou- Yokadouma. Trois types de sols peuvent être distingués dans la localité à savoir : les sols ferralitiques rouges dérivés des roches métamorphiques qui représentent l'essentiel des sols de la zone ; les sols ferralitiques rouges dérivés des roches basaltiques ; les sols à gley ou alluviaux et les sols hydromorphes rencontrés en bordure inondable. Les formations forestières sur sol ferme appartiennent au secteur forestier semi caducifolié du domaine de la forêt dense humide semi caducifolié guinéo congolaise, caractérisé par plusieurs types de formations forestières :les forêts semi caducifoliées à Sterculiaceae et Ulmaceae, les forêts mixtes, semi caducifoliées et les forêts toujours vertes du Dja avec prédominance d'éléments des forêts caducifoliées. Parmi les principales 
essences rencontrées dans la zone, on peut citer : l'Ayous (Triplochyton scleroxylon), le Fraké (Terminalia superba), le Sapelli (Entandrophragma cylindricum), le Padouk rouge (Pterocarpus soyauxii), le Tali (Erytrophleum ivorense), le Kotibé (Nesogordonia papaverifera), le Diana Z (Celtis zenkeri), le Kossipo (Entandrophragma candollei) et le Dibetou (Lovoatrichilioides). Les formations végétales sur sols hydromorphes, constituées de forêts marécageuses inondées temporairement se retrouvent dans les zones affaissées, ainsi qu'à la périphérie des zones marécageuses. Les forêts marécageuses inondées en permanence sont caractérisées par la présence de différents types de raphia, dont les plus rencontrés sont Raphia hookeri et Raphia mombuttorum. Ces formations sont aussi caractérisées par la présence des essences, telles que le Bahia (Hallea ciliata), le Rikio (Uapaca guineensis), l'Odjobi (Xylopia staudtii) et le Nsangomo (Allamblackia floribunda).

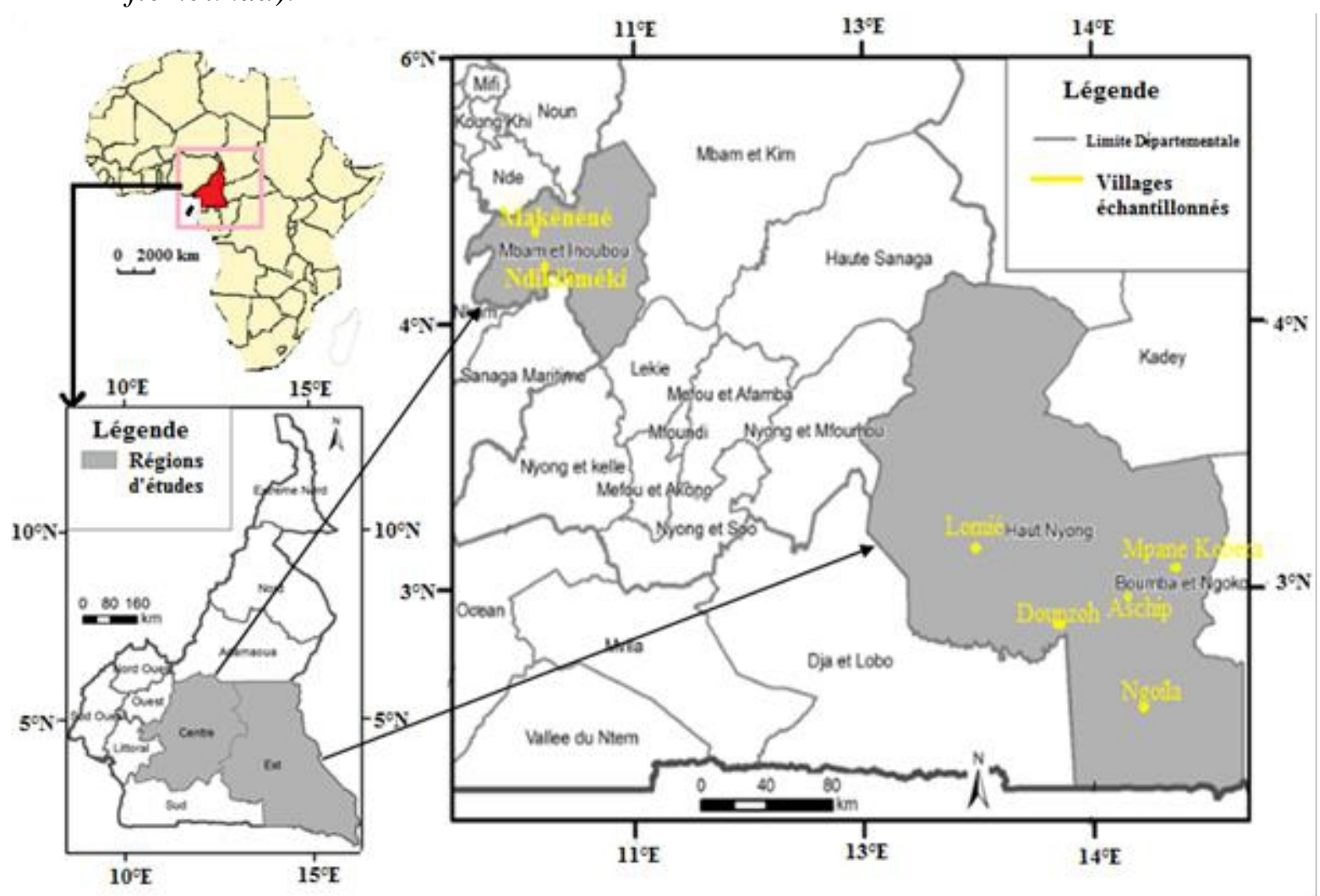

Figure 1. Localisation de la zone d'échantillonnage (villages) dans les différents régions d'études 


\section{Description, utilisations et importance économique de Garcinia kola (Clusiaceae)}

Le genre Garcinia (Clusiaceae) est largement répandu en Afrique et en Asie avec environ deux cents espèces dans ces régions tropicales (Isawumi, 1993). Des échantillons d'herbiers conservés à l'Herbier National du Cameroun indiquent la présence de vingt et une espèces de Garcinia au Cameroun. Il s'agit de G. afzelii, G. barteri, G. brevipedicellata, $G$. chromocarpa, G. elliotii, G. conrauna, G. epunctata, G. gnetoides, $G$. tinctoria, G. letestui, G. lucida, G. mangostana, G. mannii, G. ovalifolia, G. nobilis, G. polyantha, G. punctata, G. preussii, G. smeathmannii, G. staudtii et G. kola. Garcinia kola communément appelé «bitter cola » (Guttiferae/Clusiaceae; Steentoft, 1988), est un arbre de petite taille endémique de la forêt tropicale humide, des écosystèmes de l'Afrique de l'Ouest et du Centre (de la Sierra Leone à la République Démocratique du Congo). C'est une ressource socio-économiquement importante dans la sous région d'Afrique centrale et principalement pour les populations camerounaises. L'exploitation par abattage de cette espèce pour la récolte de son écorce aux vertus médicinales et comme ferment de vin de palme contribue drastiquement à sa rareté dans la région. Cette exploitation anarchique par abattage affecte la capacité de régénération naturelle de l'espèce à cause de l'absence de graines matures (Abbiw, 1990 ; Gyimah, 2000). Les graines entrent aussi dans la composition de médicaments et sont utilisées pour leurs propriétés astringentes. Légèrement acides et amères, elles sont mâchées comme substitut aux noix de colatiers (Cola spp.) et on leur reconnaît des vertus stimulantes et aphrodisiaques (Vivien \& Faure, 1996). Les parties charnues du fruit sont également comestibles. Ainsi, la surexploitation liée à la forte demande à l'échelle locale et internationale a conduit cette espèce au bord de son extinction dans son habitat naturel (Wong, 1997). L'Union Internationale pour la Conservation de la Nature (UICN) a classé Garcinia kola, dans la liste rouge des essences actuellement menacées d'extinction (UICN, 2004). Le réseau SAFORGEN a également répertorié Garcicia kola comme une espèce prioritaire pour sa conservation immédiate parmi les ressources génétiques forestières subsahariennes (Ouédraogo \& Boffa, 1999 ; Sacandé et al., 2004). Egbe et al. (2012) ont révélé que Garcinia kola figure parmi les huit espèces à PFNL (Produit Forestier Non-Ligneux) les plus importantes pour les populations de la région Sud-Ouest du Cameroun, générant des revenus situés entre environ 50 et 120 dollars US/ kg. En saison de forte production un tas d'environ 10 graines de Garcinia kola coûte 100 FCFA. Au milieu des années 1990, le kilogramme de graines de Garcinia kola coûtait en moyenne 447 FCFA (Ndoye, 1995). Ceci est comparable aux prix du cacao et du café. Ndoye et al. (1999) ont estimé sur 28 marchés dans le Sud du Cameroun une commercialisation en 1995 d'écorce de 16200 kg de 
Garcinia kola et de $40600 \mathrm{~kg}$ de Garcinia lucida. Ces quantités ont généré des montants de 3971000 FCFA et 10360000 FCFA respectivement. En 2010, Ingram et Schuré ont estimé la valeur économique annuelle de Garcinia kola au Cameroun à 249,938 US\$. Les produits de Garcinia kola exportés vers le Gabon en 1995 correspondaient à une valeur monétaire de 6,9 millions de FCFA (Ndoye et al., 1999). Les noix de Garcinia kola sont donc d'une importance considérable dans de nombreux domaines à travers l'Afrique occidentale et centrale et constituent une source supplémentaire de revenus pour la population rurale (Adepoju \& Salau, 2007).

\section{Méthodologie}

Pour l'inventaire des ligneux, l'échantillonnage a été fait par la méthode de quadrats, en s'efforçant d'intégrer les différents faciès des communautés végétales étudiées, diverses conditions naturelles et anthropiques. Chaque parcelle était constituée d'un système de quadrats emboités. Dans chaque parcelle de $1600 \mathrm{~m}^{2}$ (40 m x $40 \mathrm{~m}$ ), le diamètre des individus appartennent aux strates arborescentes $(>10 \mathrm{~m}$ de hauteur) et arbustives (2-10 $\mathrm{m}$ de hauteur) à $\mathrm{dhp} \geq 10 \mathrm{~cm}$ a été mesuré à l'aide d'un ruban circonférenciel. Ces individus ont été identifiés et recensés. Des échantillons des espèces non identifiées sur le terrain ont été récoltés et comparés à ceux de l'Herbier National de Yaoundé-Cameroun en vue de leur identification. De plus concernant $G$. kola, dans chaque relevé de $40 \mathrm{~m}$ x $40 \mathrm{~m}$, nous avons identifié et compté tous les individus, y compris les plantules. Les mensurations du dhp pour les individus ayant une dhp $\geq 10 \mathrm{~cm}$ ont été faites. La hauteur des individus de $G$. kola dans chaque relevé a été estimée visuellement après plusieurs essais réalisés avec le dendromètre Suunto (Ouédraogo, 2008 ; Agali, 2009). Un quadrat de $1 \mathrm{~m}^{2}$ a été établi autour des arbres matures afin de dénombrer les plantules présentes dans ces relevés et de mieux appréhender la capacité de régénération de la population. Pour l'ensemble des individus à $\mathrm{dhp} \geq 10 \mathrm{~cm}$ de chaque région, plusieurs paramètres floristiques ont été calculés à l'aide du tableur Excel pour caractériser le peuplement étudié, il s'agit de l'abondance relative, la fréquence, la dominance et la densité (Onana \& Devineau, 2002).

- L'abondance des taxons nous renseigne sur le nombre d'individus d'une espèce ou d'une famille sans tenir compte de la taille. Elle nous permet de calculer la densité relative des taxons (\%) et s'obtient par le rapport du nombre d'individus d'une espèce ou d'une famille en rapport au nombre total d'individus dans l'échantillon.

- La fréquence, encore appelée effectif ou répétition marque le nombre de fois qu'un taxon apparaît dans une parcelle. Elle donne ainsi une idée sur le comportement social de ce taxon et sa répartition dans 
l'espace. La fréquence relative est le rapport exprimé en pourcentage du nombre de relevés ou le taxon est présent sur le nombre total de relevés.

- La dominance d'un taxon fait appel au pourcentage de surface terrière $\left(\mathrm{G}=\square \square \mathrm{Di}^{2} / 4\right.$, où $\mathrm{D}$ est le diamètre à hauteur de poitrine) occupé par les individus appartenant à ce taxon et s'exprime en $\mathrm{m}^{2} /$ ha. Elle tient compte de la taille des individus et permet de mettre en évidence les taxons qui occupent le plus de place dans la végétation. Elle exprime ainsi la proportion de la surface terrière d'une espèce ou d'une famille par rapport à la surface terrière totale.

- La densité d'une espèce est le nombre d'individus de cette espèce à l'hectare. Elle est évaluée par la formule $\mathrm{N}=\mathrm{n} / \mathrm{S}$ (avec $\mathrm{N}$ : densité (en tiges/ha), $\mathrm{n}$ : nombre de tiges présentes sur la surface considérée, $\mathrm{S}$ : surface considérée (ha).

L'ANOVA a été utilisée pour comparer les moyennes de densité des différents relevés et voir s'il existe une différence significative et le test de DUNCAN au seuil de signification 5\% (Logiciel XLSTAT) a été utilisé pour séparer ces moyennes.

\section{Résultats et discussion}

\section{Diversité floristique des peuplements à Garcinia kola}

Les 39 relevés, soit une surface de 5,76 hectares comprennent au total 196 espèces dont les individus ont une $\mathrm{dhp} \geq 10 \mathrm{~cm}$, ces espèces se répartissent dans 124 genres et 46 familles. Parmi ces familles, 16 comptent au moins 4 espèces (Tableau 1). Les autres familles sont représentées par 3, 2 où une espèce. Il s'agit par exemple des Ochnaceae (3), des Burceraceae (3), des Olacaceae (3), des Ebenaceae (3), des Pandaceae (3), des Malvaceae (2), des Rhamnaceae (2), des Huaceae (2), des Lecythidaceae (1), des Passifloraceae (1) et des Scytopetalaceae (1) etc...Parmi les 196 espèces, G. kola est présente dans tous les 39 relevés, soit une fréquence relative de $100 \%$. La fréquence relative et l'abondance de ces familles varie en fonction des localités des différentes régions.

Tableau 1. Liste des familles les plus représentées (4 espèces au moins), classées par ordre décroissante dans l'ensemble de la flore des deux régions d'études $(\mathrm{dhp} \geq 10 \mathrm{~cm})$. Avec Nesp (Nombre d'espèce) ; Ar (Abondance relative) ; Fr (Fréquence relative) et Ngr le Nombre de genre.

\begin{tabular}{|c|c|c|c|c|c|c|}
\hline \multirow[t]{2}{*}{ Familles } & \multirow[t]{2}{*}{$\begin{array}{l}\text { Nombre } \\
\text { (Nesp) }\end{array}$} & \multicolumn{2}{|c|}{$\begin{array}{l}\text { Abondance relative } \\
\text { (Ar) }\end{array}$} & \multicolumn{2}{|c|}{$\begin{array}{l}\text { Fréquence relative } \\
\text { (Fr) }\end{array}$} & \multirow[t]{2}{*}{$\begin{array}{l}\text { Nombre de } \\
\text { Genres (Ngr) }\end{array}$} \\
\hline & & Est & Centre & Est & Centre & \\
\hline Euphorbiaceae & 20 & 9,93 & 2,40 & 94,73 & 20 & 9 \\
\hline Fabaceae & 15 & 3,30 & 2,12 & 94,73 & 75 & 14 \\
\hline Meliaceae & 13 & 2,52 & 9,73 & 89,47 & 20 & 6 \\
\hline
\end{tabular}




\begin{tabular}{lllllll}
\hline Sterculiaceae & 9 & 1,56 & 0,88 & 73,68 & 65 & 5 \\
Moraceae & 9 & 10,88 & 0,79 & 63,15 & 75 & 4 \\
Anacardiaceae & 8 & 1,73 & 0,66 & 42,10 & 45 & 6 \\
Annonaceae & 8 & 4,39 & & 63,15 & & 5 \\
Rutaceae & 8 & 1,22 & 0,84 & 21,05 & 30 & 2 \\
Apocynaceae & 8 & 6,37 & 2,39 & 73,94 & 5 & 6 \\
Rubiaceae & 7 & 1,51 & 6,27 & 42,10 & 10 & 7 \\
Ulmaceae & 7 & 1,92 & 3,84 & 78,94 & 20 & 3 \\
Caesalpiniaceae & 6 & 3,50 & 0,83 & 63,15 & 10 & 5 \\
Clusiaceae & 6 & 2,77 & 9,65 & 100 & 100 & 3 \\
Mimosaceae & 5 & 4,57 & 2,1 & 73,68 & 85 & 2 \\
Myristicaceae & 4 & 4,38 & 2,55 & 63,15 & 30 & 4 \\
Sapindaceae & 4 & 1,02 & 12,5 & 42,10 & 5 & 4 \\
\hline
\end{tabular}

Dans la région de l'Est, $G$. kola est la seule espèce présente dans tous les 19 relevés établis. Sept espèces soit 4,7\% du total des espèces ont une fréquence relative supérieur à $50 \%$, elles sont donc présentes dans 10 relevés au moins. Il s'agit de Pentaclethra macrophylla, Musanga cecropioides, Tabernaemontana crassa, Celtis tessmannii, Santiria trimeria, Petersianthus macrocarpa et Rinorea grandifolia. Quarante-neuf (49) espèces, soit 31,41 \% du total des espèces sont présentes dans un seul relevé, cela correspond à une fréquence relative inférieure à $6 \%$, il s'agit entre autres de Afrostyrax kamerunensis, Diospyros crassiflora, Harungana madagascariensis, Diospyros crassiflora, Allanblackia klaineana, Antrocaryon micraster etc.... Par contre dans les localités de Makénéné-Ndikinimeki (région du Centre), deux espèces (Garcinia kola et Dacryodes edulis), soit 2,94 du total des espèces recensées dans cette zone sont présentes dans tous les 20 relevés, soit une fréquence relative de $100 \%$. Deux espèces, ont une fréquence relative supérieure à $50 \%$, elles sont présentes dans 10 relevés au moins, il s'agit de Rauvolfia vomitoria et Ficus exasperata. Quatorze (14) espèces, soit 20,58 \% du total des espècessont présentes dans un seul relevé, cela correspond à une fréquence relative inférieure à 6\%, il s'agit entre autres de Afzelia bipindensis, Allophylus bullatus, Carapa procera, Nauclea diderrichii, Pycnanthus angolensis, Vitex grandifolia etc... Les autres espèces $(73,53 \%)$ ont une fréquence relative qui varie entre 6 et $50 \%$.

Les formations à Garcinia kola étudiées dans les régions de l'Est et du Centre Cameroun montrent une forte diversité spécifique avec respectivement 156 espèces $\left(H^{\prime}=2,98\right)$ et 68 espèces $\left(H^{\prime}=2,13\right)$ recensées. Cependant, ces formations sont peu comparables sur le plan floristique ; elles ne comptent que 34 espèces en commun. Certaines espèces sont cicatricielles, par exemple Tabernaemontana crassa, Barteria fustilosa, d'autres sont des espèces pionnières. Parmi ces dernières on peut citer Musanga cecropioides, Macaranga asas, Macaranga monodora, Alstonia boonei et Rauvolfia 
vomitoria. La proportion des espèces pionnières montre que le milieu a été perturbé et notamment par les activités anthropiques. En effet, les changements d'utilisation des terres favorisent le développement d'espèces à croissance rapide.Ce type de perturbation induit en effet une prénétration plus importante de la lumière dans le sous bois et facilite l'installation des espèces pionnières héliophiles. La reconstitution de la végétation va être donc dépendante du type de perturbation, elle est aussi fonction de la banque de graines dans le sol, de la disponibilité de l'eau et nutriments du sol. Elle peut persister plusieurs décennies avant de voir progressivement s'installer les espèces de forêt primaire (Calderon-Aguilera et al., 2012). La fréquence/présence d'espèces cicatricielles dans les formations à G. kola contribuent à la reconstitution de la structure verticale de la forêt et indiquent leur dissémination à la faveur des troués forestières (Ngueguim, 2013). Cette présence de nombreuses espèces ayant des affinités avec le sous bois forestier indique l'installation d'une forêt secondaire qui est une étape de régénération versla biodiversité originelle (Zapfack et al., 2002). On note donc un nombre considérable d'espèces de lumière à croissance rapide, à bois mou et à faible durée de vie, caractéristique des forêts exploitées. Cela montre bien que, l'exploitation forestière a entraîné une modification de la densité de certaines espèces. Il s'agit de taxons reconnues comme faisant partie généralement du cortège floristique qui participe à la régénération des forêts perturbées. Ainsi Musanga cecropioides et Myrianthus arboreus dont la présence est bien marquée ont été reconnus au Sud de la Côte-d'Ivoire comme étant des espèces très actives dans le processus de la cicatrisation (Alexandre, 1989).

Il ressort également de l'analyse des différents relevés que l'abondance des espèces varie en fonction des localités dans les régions. Dans les localités de l'Est, trois espèces (Musanga cecropioides, Rinorea grandifolia et Afrostyrax lepidophyllus) soit 1,9\% du nombre total des espèces recensées ont une abondance relative supérieure à $5 \%$, abondance calculée sur la basede l'ensemble des individus recensés dans cette région. Vingt-et-neuf (29) espèces $(18,58 \%)$ ont une abondance relative comprise entre 1 et $2 \%$, il s'agit de Pentaclethra macrophylla, Coelocaryon preussii, Tabernaemontana crassa, Uapaca paludosa, Hylodendron gabunense, Garcinia kola, Plagiostyles africana, Bridelia speciosa, Santiria trimeria, Petersianthus macrocarpa, Margaritaria discoidea, Albizia adianthifolia, Greenwayodendron suaveolens etc. Dans cette zone 124 espèces, soit 79,48 $\%$ des espèces ont une abondance relative inférieure à $1 \%$. Il s'agit entre autres de Pynanthus angolensis, Allablackia floribundus, Annonidium manni, Cola lateritia, Heisteria parvifolia, Ricinidendron heudelotii, Alstonia boonei, Baillonelala toxisperma, Hymenocardia lirata, Anthonotha macrophylla, Entandropragma cylindricum. Le reste des espèces identifiées dans cette zone ont une abondance relative beaucoup plus faible $(\leq 0,5 \%)$. 
Il ressort également de l'analyse des différents relevés que l'abondance des espèces varie en fonction des localités dans les régions. Dans les localités de l'Est, 12 soit 7,69 \% du nombre total des espèces recensées ont une abondance relative beaucoup plus élevée $(2 \% \leq \mathrm{Ar} \leq 7,5 \%)$, abondance calculée sur la base de l'ensemble des individus recensés dans cette région (Tableau 2). Quarante (40) espèces ont une abondance relative comprise entre 0,5 et $2 \%$. Les autres espèces (104), soit $66,67 \%$ des espèces ont une abondance relative inférieure à $0,5 \%$. Il s'agit entre autres de Pycnanthus angolense, Allanblackia floribunda, Annonidium manni, Cola lateritia, Heisteria parvifolia, Ricinidendron heudelotii, Alstonia boonei,Baillonelala toxisperma, Hymenocardia lirata, Anthonotha macrophylla, Entandropragma cylindricum etc... Le reste des espèces identifiées dans cette zone ont une abondance relative beaucoup plus faible $(\leq 0,5 \%)$. Tout comme dans la région de l'Est, 12 espèces recensées dans la région du Centre ont une abondance relativement élevée $(1,23 \leq \mathrm{Ar} \leq 30,60)$ (Tableau 2$)$. Vingt-et-cinq (25) espèces soit $36,76 \%$ des espèces ont une abondance relative qui varie entre 0,5 et 1,15\%. Il s'agit de Musanga cecropioides, Ceiba pentandra, Lannea welwitschii, Homalium letestui, Pterocarpus soyauxii, Citrus sinensis, Cola lateritia, Lophira alata, Myrianthus arboreus, Ricinodendron heudelotii, Tetrapleura tetraptera, Ficus etrangula, Ficus mucoso, Mansonia altissima, Markhamia lutea, Nesogordonia papaverifera, Holoptelea grandis, Margaritaria discoidea et Carapa procera etc...Le reste des espèces (31) recensées dans cette zone ont une abondance beaucoup plus faible (inférieur à $0,5 \%)$.

Tableau 2. Abondance relative des 10 espèces les plus représentées dans les peuplements à G. kola en fonctions de localités échantillonnées.

\begin{tabular}{|c|c|c|c|}
\hline $\begin{array}{l}\text { Régions } \\
\text { d'études }\end{array}$ & Espèces & $\begin{array}{c}\text { Abondance } \\
\text { relative (Ar) }\end{array}$ & $\begin{array}{c}\text { Fréquence } \\
\text { relative }(\mathbf{F r})\end{array}$ \\
\hline \multirow{12}{*}{ Centre } & Dacryodes edulis & 30,58 & 100 \\
\hline & Garcinia kola & 21,76 & 100 \\
\hline & Theobroma cacao & 5,25 & 35 \\
\hline & Persea americana & 2,79 & 5 \\
\hline & Albizia adianthifolia & 2,68 & 30 \\
\hline & Ficus exasperata & 2,12 & 50 \\
\hline & Albizia zygia & 1,90 & 25 \\
\hline & Terminalia superba & 1.90 & 40 \\
\hline & Cordia africana & 1,79 & 25 \\
\hline & Distemonanthus benthamianus & 1,56 & 40 \\
\hline & Pterygota bequaertii & 1,34 & 15 \\
\hline & Cola accuminata & 1,23 & 15 \\
\hline & Musanga cecropioides & 7,07 & 63,16 \\
\hline & Rinorea grandifolia & 5,15 & 52,63 \\
\hline & Afrostyras lepidophyllus & 5,0 & 42,11 \\
\hline & Myrianthus arboreus & 2,84 & 36,84 \\
\hline & Pentaclethra macrophylla & 2,84 & 68,42 \\
\hline
\end{tabular}




\begin{tabular}{|c|c|c|c|}
\hline \multirow{4}{*}{ Est } & Coelocaryon preussii & 2,53 & 42,11 \\
\cline { 2 - 4 } & Tabernaemontana crassa & 2,53 & 63,16 \\
\cline { 2 - 4 } & Uapaca palidosa & 2,38 & 47,37 \\
\cline { 2 - 4 } & Hylodendron gabunense & 2,30 & 42,11 \\
\cline { 2 - 4 } & Bridelia speciosa & 2,07 & 31,58 \\
\cline { 2 - 4 } & Garcinia kola & 2 & 100 \\
\cline { 2 - 4 } & Plagiostyles africana & 2 & 26,32 \\
\hline
\end{tabular}

L'analyse de la composition floristique des faciès de G. kola montre que 41 familles ont été recensées dans 39 relevés, soit 36 familles à l'Est et 29 dans les localités du Centre. Cette composition varie en fonction des régions, les deux régions n'ont en commun que 21 familles. Dans la région de l'Est, la famille des Clusiaceae est présente dans tous les relevés, ce qui correspond à une fréquence relative de $100 \%$. Quinze (15) familles sont présentes dans 10 relevés au moins, soit une fréquence relative supérieure à $50 \%$, il s'agit des Euphorbiaceae, des Fabaceae, des Meliaceae,des Ulmaceae, des Apocynaceae, des Mimosaceae, des Sterculiaceae, des Annonaceae, des Caesalpiniaceae, des Moraceae, des Myristicaceae, des Violaceae, des Burceraceae, des Irvingiaceae et des Lecythidaceae. Deux de ces familles, soit $5,55 \%$ du total des familles rencontrées à l'Est ne sont présentes que dans un seul relevé, leur fréquence relative est donc inférieure à $6 \%$; il s'agit des Chrysobalanaceae et des Myrtaceae. Dans la région du Centre, deux familles (Clusiaceae, Burseraceae) sont présentes dans tous les relevés, ce qui correspond à une fréquence relative de $100 \%$. Cinq familles sont présentes dans 10 relevés au moins, ce qui correspond à une fréquence relative supérieure à $50 \%$, il s'agit des Mimosaceae, des Fabaceae, des Moraceae, des Sterculiaceae et des Rutaceae. Cinq familles, soit 17,24\% des 29 familles récencées dans cette région ne sont présentes que dans un seul relevé. Leur fréquence relative est donc inférieure à $6 \%$; il s'agit des Apocynaceae, des Lecythidaceae, des Sapindaceae et des Verbenaceae. Les Euphorbiaceae, Fabaceae, Meliaceae, Malvaceae, Moraceae, Anacardiaceae, Apocynaceae, Annonaceae, Rutaceae, Rubiaceae, Ulmaceae, Caesalpiniaceae, Clusiaceae et les Mimosaceae recencées dans les deux régions sont les plus représentées dans les formations à G. kola. Selon Xiao et al. (2010), les Fabaceae, les Euphorbiaceae, les Annonaceae et les Rubiaceae sont des familles communes des forêts tropicales. D'autres études menées dans la réserve de Dja (Cameroun) sur la diversité floristique ont montré que les Fabaceae, les Rubiaceae, les Malvaceae, les Euphorbiaceae, les Sapotaceae, les Annonaceae et les Meliaceae sont les familles les plus diversifiées (Sonké \& Couvreur, 2014).

Les familles montrant les plus fortes abondances pour les individus à dhp $\geq 10 \mathrm{~cm}$ varient en fonction des localités. Dans les localités de la région de l'Est, les Moraceae et les Euphorbiaceae sont les plus abondants, soit 
$10,88 \%$ et $9,33 \%$ respectivement du nombre total des individus recencés. Cinq autres familles ont une abondance relative supérieure à $4 \%$, il s'agit des Huaceae, des Mimosaceae, des Annonaceae, des Myristicaceae et des Violaceae. Vingt-sept familles ont une abondance relative comprise entre 0,70 et 3,5\%, il s'agit des Caesalpiniaceae, des Fabaceae, des Olacaceae, des Burceraceae, des Meliaceae, des Hymenocardiaceae, des Clusiaceae, des Lecythidaceae etc. Dans les localités du Centre, les Malvaceae et les Burseraceae sont les plus abondantes, avec une abondance relative de 15,38\% et $15,25 \%$ respectivement. Cinq familles ont une abondance supérieure à $5 \%$, il s'agit des Sapindaceae, des Apocynaceae, des Meliaceae, des Clusiaceae et des Rubiaceae. Vingtetune (21) familles présentes dans ces localités ont une abondance relative comprise entre 0,66 et 3,87\%, il s'agit des Lauraceae, des Lecythidaceae, des Ulmaceae, des Malvaceae, des Flacourtiaceae, des Verbenaceae, desLecythidaceae, des Euphorbiaceae etc...

La dominance fait appel au pourcentage de surface terrière occupé par les individus appartenant à la famille et s'exprime en $\mathrm{m}^{2} / \mathrm{ha}$. Cette dominance varie aussi en fonction des localités dans les deux régions. Dans la région de l'Est, une seule famille a une dominance relative supérieure à $10 \%$, il s'agit des Pandaceae (12,74\%). Six autres familles ont une dominance relative comprise entre 5 et $10 \%$, il s'agit des Irvingiaceae, des Mimosaceae, des Huaceae, des Sapotaceae, des Malvaceae et des Fabaceae. Vingt-et-huit familles ont une dominance relative inférieure à 5\%, il s'agit des Myriscaceae, des Rutaceae, des Combretaceae, des Caesalpinaceae, des Clusiaceae, des Ulmaceae, des Meliaceae, des Lecythidaceae, des Olacaceae etc...Dans la région du Centre, les Malvaceae sont la seule famille ayant une dominance relativement élévée $(41,53 \%)$. Cinq autres familles ont une dominance relative comprise entre 5 et 9\%, il s'agit des Euphorbiaceae, des Boraginaceae, des Caesalpinaceae, des Ulmaceae et des Lecythiaceae. Vingt-deux (22) familles ont une dominance relative inférieure à 5\%, il s'agit des Ulmaceae, des Ochnaceae, des Combretaceae, des Fabaceae, des Bignoniaceae, des Burseraceae, des Mimosaceae, des Clusiaceae, des Verbenaceae, des Salicaceae etc...

\section{Densité des individus par espèce dans les formations à base de Garcinia kola}

L'analyse de la densité des individus par espèce dans les formations à G. kola dans les différentes localités de la région de l'Est montre que Musanga cecropioides (33,55 tiges/ha) a une densité des individus à $\mathrm{dhp} \geq 10 \mathrm{~cm}$ largement supérieure celles des autres espèces dans les localités de la région de l'Est. Elle est suivie par Afrostyrax lepidophyllus (24,33 tiges/ha), Rinorea grandifolia (19,07 tiges/ha) et Pentaclethra macrophylla (15,79 tiges/ha). Dans ces localités, Garcinia kola a une densité moyenne de 5,26 tiges par 
hectare. Les autres espèces identifiées ont une densité comprise entre 8,22 et 0,33 tiges par hectare. Il s'agit par exemple de Trichilia rubescens, Plagiostyles africana, Macaranga monodora, Uapaca palidosa, Santiria trimeria, Bridelia speciosa, Celtis tessmannii, Pynanthus angolensis, Staudtia kamerouniensis, Allanblackia floribunda et Pterocarpus soyauxii. Par contre, dans les sites échantillonnées dans le Centre, Dacryodes edulis a la densité la plus élevé (73,13 tiges/ha), suivie de Garcinia kola (54,18 tiges/ha), Theobroma cacao (15 tiges/ha) et Albizia adiantifolia (6,87 tiges/ha). Les autres espèces ont des densités comprises entre 4,88 et 0,31 tiges par hectare. Il s'agit entre autres de Terminalia superba, Distemonanthus benthamianus, Myrianthus arboreus, Sterculia rinopelata, Triplochiton scleroxylon, Ficus mucoso, Pterygota bequaertii, Margaritaria discoidea, Celtis zenkeri, Ricinodendron heudelotii, Spathodea campanulata et Ceiba pentandra.

La densité moyenne d'arbres à $\mathrm{dhp} \geq 10 \mathrm{~cm}$ pour l'ensemble des peuplements à $G$. kola de la région de l'Est est beaucoup plus élevée $(576,31$ tiges/ha) que celle obtenue au Centre (307,81 tiges par hectare). Ces valeurs sont dans tous les cas inférieures à celles obtenues par Ngueguim (2013) dans les plantations forestières de Mangombé (708 tiges/ha) et par Ukizintambara et al. (2007) dans la station forestière de Lopé au Gabon (741,5-931,5 tiges/ha). La densité obtenue dans la région de l'Est est cependant supérieure à celles obtenues par Djuikouo et al. (2010) dans la réserve du Dja (350-460,4 tiges/ha), par Parmentier et al. (2011) dans la station forestière de Korup (Cameroun; 492 tiges/ha) et par Ngueguim (2013) dans les plantations forestières de Campo (569 tiges/ha) et Bidou (538 tiges/ha). Elles sont cependant comparables à celles d'autres travaux ménés dans la zone tropicale, il s'agit des études menées par Guedje (2002) dans les formations à Garcinia lucida au Sud Cameroun (629 tiges par hectare) et par Momo et al. (2017) dans les formations à Gnidia glauca au mont Oku (Nord-Ouest) où la densité moyenne était de 321,6 tiges par hectare. La faible densité observée dans le Centre s'expliquerait principalement par l'exploitation humaine, car les localités de cette région sont des grands bassins agricoles. La création de nouvelles plantations et 1'exploitation forestière à différentes échelles, engendreraient la destruction progressive des forêts naturelles au profit des formations anthropisées où la diversité et la structure des peuplements peuvent être significativement différentes en comparaison aux formations d'origine. Les activités anthropiques peuvent en effet modifier de façon significative les paramètres de diversité et de structure des peuplements végétaux tels que la densité, la surface terrière, la diversité floristique et la distribution géographique des taxons comme l'ont montré un certain nombre d'auteurs (Puig, 2001 ; Lourmas, 2003 ; Senterre, 2005 ; Thapa et al., 2010 ; Naing et al., 2011). Ces pressions ont en effet une influence directe sur l'intensité lumineuse qui pénètre dans le sous bois (Dupuy \& Chazdon, 2006) et 
corrélativement sur la densité du peuplement, la diversité et même la composition spécifique (Hitimana et al., 2004; Kessler et al., 2005). Néanmoins, l'interaction biologique liée aux conditions climatiques, édaphiques et l'écologie de chacune des espèces, sont les paramètres qui peuvent également expliquer la variation de la densité au sein des peuplements d'une région à l'autre (Martinez-Meyer, 2015 ; Illoldi-Rangel \& Escalante, 2008). La densité moyenne pour l'ensemble du peuplement étudié (441,9 tiges/ha) se situe dans l'ordre de grandeur des densités moyennes des peuplements en forêt tropicale humide estimée à 400-650 individus de dhp > $10 \mathrm{~cm}$ par hectare (Gentry, 1988).On peut donc penser globalement qu'en dépit des activités anthropiques et des perturbations d'origine naturelle dans ces formations forestières, elles se reconstituent rapidement. Cela est perceptible sur le terrain par la présence de nombreux recrûs forestiers dans les sites étudiés.

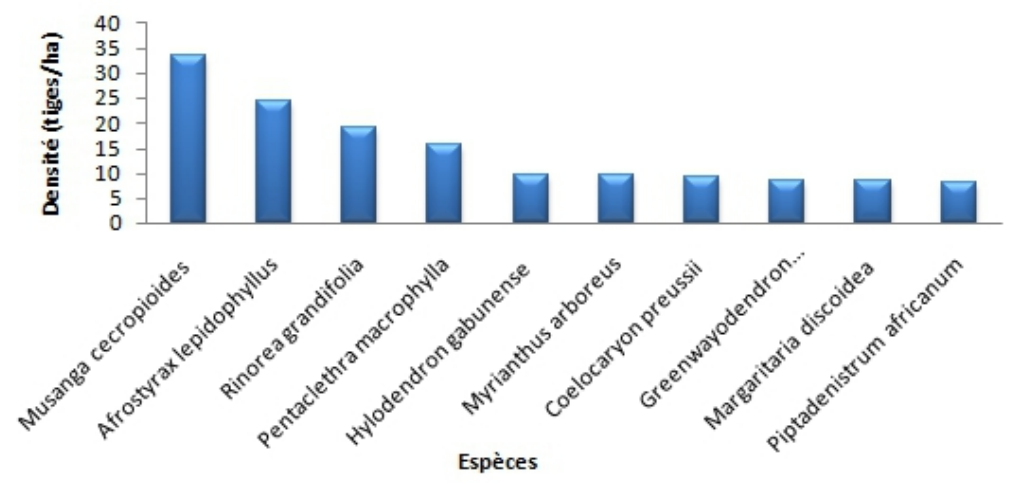

(a)

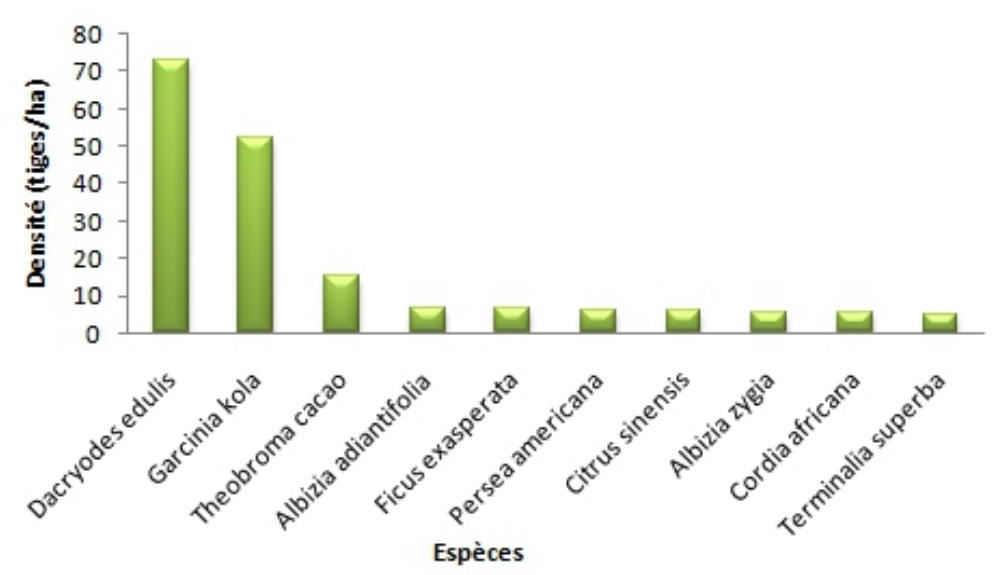

(b)

Figure 2. Densité moyenne des dix espèces les plus représentées (> 5tiges/ha) dans les formations de G. kola dans les différents localités des Régions de l'Est (a) et du Centre (b) 


\section{Analyse des populations de Garcinia kola}

La répartition des individus en classes de hauteur ou de diamètre est considérée comme un indicateur indirect du niveau d'équilibre des classes d'âge et peut rendre compte des phases vécues par la population en termes de perturbation et de régénération, et d'autre part, peut indiquer si l'intensité de l'exploitation a affectée la structure du peuplement dans les zones étudiées (Onana \& Devineau, 2002 ; Momo et al.,2017).

\section{Distribution des individus par classes de hauteur}

L'un des principaux critères permettant d'évaluer la dynamique d'une espèce ou d'un peuplement est son aptitude à compenser la mortalité par le recrutement de nouveaux individus pour maintenir sa population (Peters, 1994). Plus cette stratégieest efficace, plus longtemps la population pourra se maintenir dans l'écosystème. La répartition des individus de G. kola en fonction des classes de hauteur dans les différents sites d'échantillonnage au Centre et à l'Est du Cameroun montre une faible représentativité des jeunes individus dans tous les sites, ce nombre de jeunes individus est toutefois relativement plus élevé à Makénéné et Ndikinimeki par rapport aux autres sites (Figure 3). Globalement, les plus grands effectifs par classe de hauteur se retrouvent dans les localités du Centre (Makénéné et Ndikinimeki).On peut ainsi noter que dans la classe de hauteur variant entre 0 et $5 \mathrm{~m}$ les plus grands effectifs (> 30 individus) se retrouvent dans ces deux localités. De même, pour 1'ensemble des classes de hauteur supérieure à $10 \mathrm{~m}$, l'efffectif des individus est plus élevé à Makénéné (39 individus), suivi de Ndikinimeki (39 individus). Entre 2 et $10 \mathrm{~m}$ de hauteur, on note un quasi absence des jeunes individus dans les localités comme Dounzoh, Ngoïla, Mpane Kobera et Aschi (Est) alors que 1'effectif global dans les localités du Centre (Makénéné et Ndikinimeki) est de 124 individus. Cette faible proportion des jeunes individus dans les localités de l'Est peut s'expliquer par une forte pression anthropique à travers différentes formes d'exploitation des individus dans cette région du Cameroun qui ne s'accompagne pas de pratiques favorables à la régénération de l'espèce. Par contre, la forte proportion des jeunes individus dans les localités du Centre pourrait s'expliquer par des conditions climatiques et édaphiques, par une structure de la végétation plus favorable à la germination des graines et surtout par une prise de conscience des exploitants pour gérer de façon durable la ressource. Cette zone est en effet caractérisée par une canopée moins fermée, qui autorise une meilleure pénétration de la lumière dans le sous bois, conditions nécessaire à une bonne germination. Des études menées dans les forêts tropicales ont montré toutefois que $G$. kola tolère l'ombre et pousse donc sous les forêts à canopée fermée où les conditions météorologiques et les niveaux d'humidité du sol sont relativement stables (Hall \& Swaine, 1981). Mais des observations sur le terrain nous ont permis de relativiser cette 
donnée ; on constate en effet que l'ouverture des trouées pourrait favoriser la germination et même augmenter la productivité des pieds de bitter kola. Selon Aiyelaagbe et al. (1996), ces arbres sont abondants dans les zones densément peuplées de forêts naturelles et secondaires où le système prédominant d'utilisation des terres est la plantation d'arbres et de cultures. La présence d'un grand nombre des jeunes individus chez une espèce témoigne d'une bonne capacité de régénération telle que cela a été observé chez certaines espèces comme Clausena anisata (Kémeuzé et al., 2009) et Gnidia glauca (Momo et al., 2017) dans la région du Nord-Ouest (Cameroun). La faible représentativité des individus de grande taille à l'Est peut s'expliquer par la pression exercée sur la ressource dans le milieu naturel depuis plusieurs décennies. En effet, l'exploitation par abattage de cette espèce pour la récolte de son écorce aux vertus médicinales et comme ferment de vin de palme contribue la réduction drastique des effectifs et doncà sa rareté dans la région de l'Est. Cette exploitation anarchique des adultes par abattage affectent la capacité de régénération naturelle de l'espèce car en l'absence de semenciers les graines matures deviennent de plus en plus rares (Abbiw, 1990 ; Gyimah, 2000).

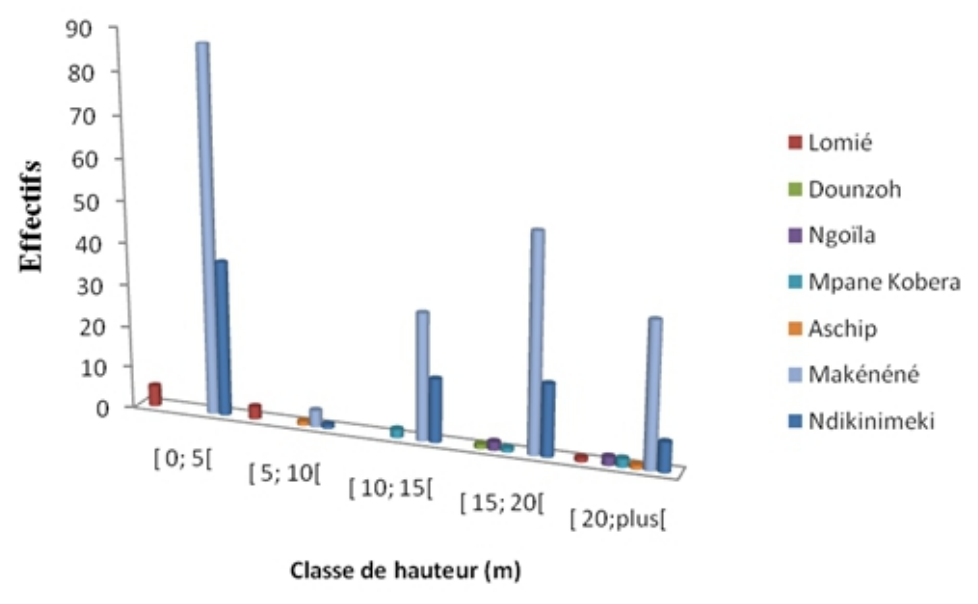

Figure 3. Distribution des individus par classes de hauteur chez Garcinia kola dans les différents sites d'échantillonnage

\section{Distribution par classes de diamètre des populations de Garcinia kola}

La distribution des individus par classes diamètre dans les différentes régions montre une faible proportion des individus de faible diamètre ([0-10 $\mathrm{cm}]$ ) dans plusieurs localités aussi bien de l'Est que du Centre à l'exception de Makénéné et Ndikinimeki (Figure 4). Ces deux localités du Centre montrent en effet un nombre d'individus relativement élevé dans cette classe de diamètre ( 27 et 12 individus respectivement). Les plus faibles valeurs étant rencontrées dans les localités de Ngoïla et Mpane Kobera à l'Est. Entre 10 à $>20 \mathrm{~cm}$ de diamètre, on observe également dans les localités de Makénéné et 
Ndikinimeki, un nombre d'individus plus elevé comparé aux autres localités (57 et 18 individus). A partir de cette classe de diamètre, le nombre d'individus décroit progressivement pour atteindre logiquement les valeurs les plus faibles dans la classe de diamètre entre $50 \mathrm{~cm}$ et $>50 \mathrm{~cm}$. Les localités de l'Est, et notamment Ngoïla et Mpane Kobera montrent une légèrement augmentation des individus dans les classes de diamètre $>40 \mathrm{~cm}$ mais les valeurs restent globalement faibles comparées à Ndikinimeki et Makénéné. Le fait qu'à l'exception de deux sites (Ngoïla et Mpane Kobera), les effectifs de la classe de diamètres compris entre 10 et $20 \mathrm{~cm}$ soient supérieurs à ceux de la classe de diamètre 0 et $10 \mathrm{~cm}$ indique probablement une perturbation récente qui peut être d'origine anthropique ou climatique. Cela peut ainsi être lié à l'exploitation de la ressource pour divers produits, et surtout par les méthodes d'exploitation observées dans cette zone (annélétion, abattage et déracinement). Ces méthodes destructrices provoquent en effet la mort des individus exploités et à long terme peuvent entrainer la rareté des individus de l'espèce (Guedje, 2002). Cette pression semble aussi avoir été encore plus importante dans les classes de diamètre entre 20 et $40 \mathrm{~cm}$ dans l'ensemble des sites à l'excepion de Makénéné et Ndikinimeki. La pression exercée dépend en effet de l'utilité de la ressource pour la population locale, mais aussi de la disponibilité de la ressource. Par exemple, des travaux menés dans la zone de Bipindi-Akom II (Sud-Cameroun) ont montré que le lieu de collecte des Produits Forestiers Non Ligneux est étroitement en relation avec leur disponibilité qui elle-même est menacée par les techniques de récoltes et les parties de l'arbre utilisées. Ces études ont en effet montré que certaines espèces comme Baillonella toxisperma, Garcinia lucida et surtout le Garcinia kola sont les espèces dont la disponibilité du potentiel est couramment en baisse. Les techniques de récolte associées sont parmi les plus destructrices puisqu'il s'agit de l'écorçage et de l'abattage (Guedje, 2002). Leur disponibilité dans cette région forestière diminue au point où leurs aires de distribution autour des villages diminuent aussi. Les villageois se trouvent alors obligés de parcourir de longues distances pour trouver des individus à exploiter. Yemadje (2011), pense que la forte réduction de l'effectif des individus de classes de diamètre supérieures montre que le peuplement étudié a probablement subi une perturbation, liée à l'exploitation de plusieurs produits. Dans les sites de Makénéné et Ndikinimeki, à partir de la classe entre 10 et $20 \mathrm{~cm}$ on observe une diminution progressive du nombre d'individus au fur et à mesure qu'on évolue vers les gros diamètres, ce qui est caractéristique des peuplements à régénération constante dans le temps et de formations végétales en équilibre. Cette structure forestière est semblable à celle de Rollet (1974), qui a montré que la distribution des classes de diamètre des arbres suit un modèle exponentiel. En dépit de cette structure quasi normale à partir de $10 \mathrm{~cm}$ de diamètre, il faut néanmoins noter un déficit de grands arbres dans les 
différentes localités qui s'expliquerait par l'exploitation sélective par la population riveraine.Cela indique une forme d'exploitation différente de celle de la région de l'Est où toutes les classes de diamètre semblent affectées par l'exploitation. La distribution des arbres par classe de diamètre est d'une grande importance dans l'étude de la dynamique spatio-temporelle des peuplements, elle a en effet été utilisée par plusieurs auteurs pour donner une représentation simplifiée de la structure d'un peuplement (Onana \& Devineau, 2002 ; Ukizintambara et al., 2007 ; Chazdon et al., 2010 ; Liwen Zhang et al., 2010 ; Luna Vega et al., 2010). Elle renseigne sur la dynamique forestière et est indispensable en sylviculture, c'est un outil d'aide à la gestion forestière qui peut ainsi servir pour le cas des communautés végétales étudiés.

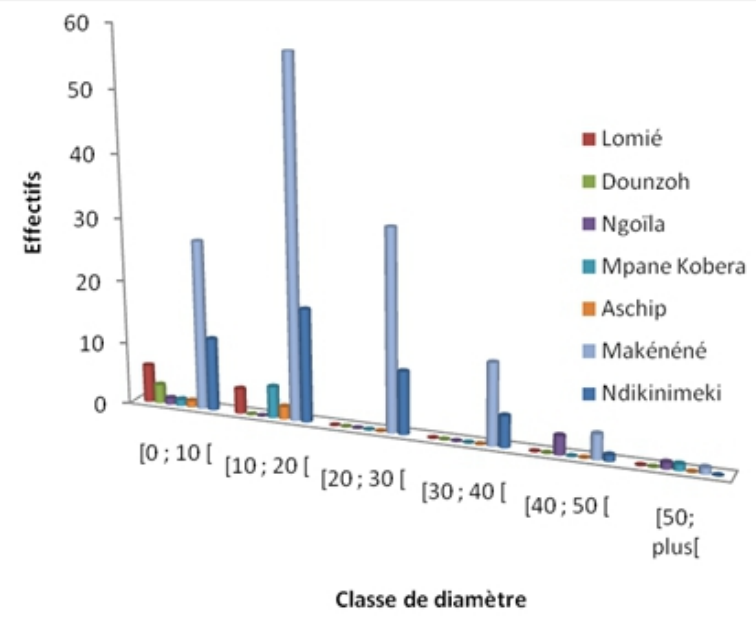

Figure 4. Distribution des individus par classes de diamètre de G. kola

\section{Densité du peuplement de $\boldsymbol{G}$. kola en fonction des sites}

La distribution des densités de la population de G. kola par classes de diamètre dans les différents sites montre une tendance comparable à celle des effectifs totaux par classes de diamètre. Elle montre qu'à l'exception des sites de Lomié et Dounzoh, les densités sont plus élevées dans la classe $10-20 \mathrm{~cm}$ par rapport à la classe $0-10 \mathrm{~cm}$ contrairement à ce qu'on se serait attendu (Figure 5). Les sites de Makénéné et Lomié montrent une densité moyenne relativement élevée $(8,13$ et 7,5 tiges/ha) par rapport aux autres sites dans la classe de diamètre [0-10 cm]. A Ngoïla et à Mpane Kobera la densité dans cette classe est la plus faible (0,34 tiges/ha). De manière générale après la classe $[0-10 \mathrm{~cm}]$, on observe une diminution de la densité avec une augmentation des classes de diamètre, ainsi que le montre l'évolution de la densité moyenne par classes de diamètre pour l'ensemble des 7 sites d'échantillonnage (Figure 5). Dans les sites de Makénéné et Ndikinimeki, à partir de $10 \mathrm{~cm}$ de diamètre, elles varient de 43,5 à 0,48 tiges par hectare en moyenne. Dans les autres sites les densités les plus faibles sont enregistrées 
entre 20 et $40 \mathrm{~cm}$ de diamètre, puis elles augmentent pour les sites de Ngoïla et Mpane Kobera pour atteindre les valeurs les plus élevées qui varient de 4,68 à 1,56 tiges/ha.

Toutefois, on peut globalement noter qu'entre 10 et $40 \mathrm{~cm}$ de diamètre, les densités moyennes sont plus élevées dans les sites de Makénéné et Ndikinimeki (49,25 et 29,49 tiges/ha) comparées à celles des autres sites. Dans la classe [40-50 cm], cette densité moyenne est plutôt plus élevée à Ngoïla (4,68 tiges/ha). Lorsqu'on considère l'ensemble des classes de diamètre, la densité moyenne est relativement plus élevée à Makenéné (59,73 tiges/ha), suivie de Ndikinimeki (35,81 tiges/ha), Lomié (13,75 tiges/ha), Mpane Kobera (9,37 tiges/ha). Les autres sites ont des densités faibles ( $\leq 6,25$ tiges/ha). Cette tendance reste la même lorsqu'on considère la densité moyenne des individus à $\mathrm{dhp} \geq 10 \mathrm{~cm}$. Il ressort en effet du tableau 1 que la densité moyenne des individus à $\mathrm{dhp}>10 \mathrm{~cm}$ est globalement faible dans les localités étudiées. Toutefois cette densité est plus élevée à Makénéné (51,60 tiges/ha), suivie de Ndikinimeki (30,44 tiges/ha), Mpane Kobera (9,37 tiges/ha), mais reste faible à Aschip (4,18 tiges/ha). L'analyse statistique (test de Duncan) montre que la densité à Makénéné (51,60 tiges/ha) est significativement élevée comparée aux autres zones d'échantillonnage (Tableau 3 ).

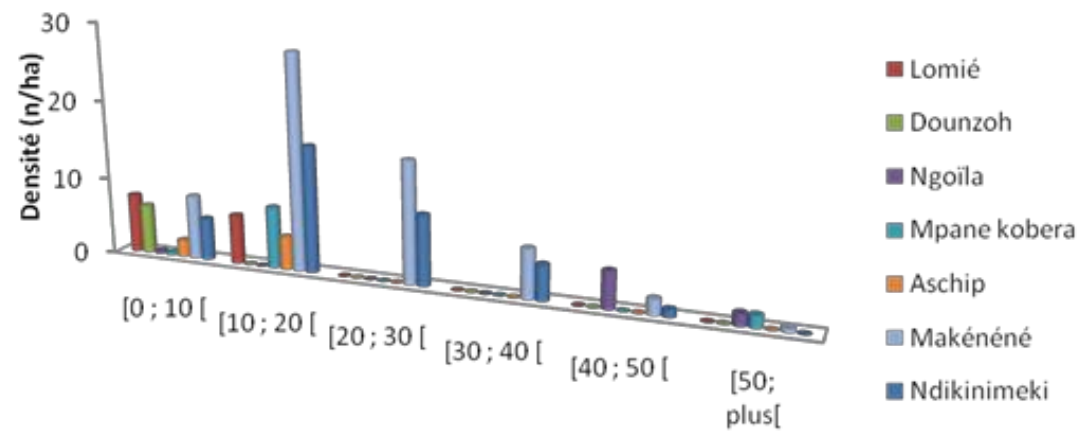

Classe de diamètre

Figure 5. Densité de G.kola dans les différents sites en fonction des classes de diamètres.

Tableau 3. Densité moyenne dans les différents sites d'échantillonnages

\begin{tabular}{lll} 
Sites & $\begin{array}{c}\text { Densité moyenne } \\
\text { estimée (tiges/ha) }\end{array}$ & $\begin{array}{l}\text { Ecart- } \\
\text { type }\end{array}$ \\
\hline Lomié & $6,25 \mathrm{~b}(\mathrm{n}=5)$ & $\pm 5,00$ \\
Dounzoh & $6,25 \mathrm{~b}(\mathrm{n}=3)$ & $\pm 0,00$ \\
Ngoïla & $6,25 \mathrm{~b}(\mathrm{n}=4)$ & $\pm 0,00$ \\
Mpane kobera & $9,38 \mathrm{~b}(\mathrm{n}=4)$ & \pm 313 \\
\hline Aschip & $4,19 \mathrm{~b}(\mathrm{n}=3)$ & $\pm 0,00$ \\
Makénéné & $51,6 \mathrm{a}(\mathrm{n}=13)$ & $\pm 32,62$ \\
Ndikinimeki & $30,44 \mathrm{ab}(\mathrm{n}=7)$ & $\pm 24,75$ \\
\hline
\end{tabular}


Les moyennes ayant les mêmes lettres ne sont pas significativement différentes au seuil de probabilité $\mathrm{P} \square 0,05$. Avec n qui est le nombre de relevés par site.

\section{Potentiel sur pied de Garcinia kola dans les differents regions}

Le potentiel sur pied de $G$. kola est le stock de tiges à dhp $\geq 10 \mathrm{~cm}$, debout sur pied et exploitable pour l'écorce (Momo et al., 2017). Ce potentiel dépend de la structure diamétrique de l'espèce et s'exprime par rapport au déploiement spatial du peuplement des formations où cette espèce montre une abondance particulière. La densité moyenne des tiges à dhp $\geq 10 \mathrm{~cm}$ varie en fonction des sites d'échantillonnage dans les différentes localités des régions étudiées. Cette valeur varie entre 6,25 à 51,60 tiges par hectare. Dans les formations à Garcinia lucida, Guedje (2002) avait obtenu des valeurs comprises entre 38 et 73 tiges par hectare, à Bipindi-Akom II (Sud Cameroun) une densité de 23 tiges/ha de G. lucida a été observée (Dijk, 1999). Certaines études similaires dans la région du Nord-Ouest (Cameroun) ont montré que la densité des populations de Gnidia glauca varie en fonction des sites échantillonnés (Momo et al., 2017). Pour ces auteurs, cette variation de la densité serait liée à la pression anthropique que subit l'espèce dans la zone. La différence observée peut également s'expliquer par les méthodes et les surfaces d'échantillonnage qui ne sont pas les mêmes. Selon Guedje (2002), les inventaires par méthode de parcelle ont des valeurs plus élevées que les inventaires par méthode de transects, car la première permet de sonder plus intensément une surface donnée (Maître, 1986; Condit, 1995). Ces différences peuvent aussi s'expliquer par la pression exercée sur chacune des espèces dans son milieu naturel. La variabilité de la densité serait liée à l'utilité des espèces pour la population locale et surtout aux modes d'exploitation. L'exploitation anarchique (abatages où déracinement) pourrait conduire à une réduction significative de la densité des individus (Guedje, 2002).

\section{Conclusion}

Cette étude menée dans les localités du Centre et de L'Est révèle une diversité végétale importante dans les formations à Garcinia kola, elles sont caractérisées par une forte diversité des espèces, des genres et des familles. Les familles les plus fréquentes, abondantes et dominantes sont les Clusiaceae, les Euphorbiaceae, les Fabaceae, les Meliaceae les Hauceae les Annonaceae, les Pandaceae, les Malvaceae et Ulmaceae. La distribution des densités de la population de G. kola par classes de diamètre dans les différents sites montre une tendance comparable à celle des effectifs totaux par classes de diamètre. Cette densité varie suivant les classes de diamètre et les sites. Dans certains cas, on peut noter très peu de plantules comparées à l'effectif des individus dans les classes de diamètre plus élevés. La densité moyenne reste plus élevée 
à Makénéné suivie de Ndikinimeki et Lomié. La faible densité observée principalement dans les localités de la région de l'Est est due à la pression exercée sur cette espèce dans son milieu naturel et notamment aux méthodes d'exploitation destructrices utilisées par la population locale. Il serait judicieux de faire une étude sur l'impact écologique de l'exploitation de l'écorce sur la structure, la dynamique de l'espèce et le taux de récupération de l'écorce de G. kola, afin de définir le quota à exploiter pour une gestion durable de la ressource.

\section{Acknowledgment}

Les auteurs remercient tous les chercheurs de l'Unité de Recherche en Botanique Appliquée pour leur collaboration et les personnes qui connaissent les produits forestiers non ligneux retrouvés dans les diverses localités échantillonnées.

\section{References:}

1. Abbiw, D.K. (1990). Useful Plants of Ghana, West African Use of Wild and Cultivated Plants.Intermediate Technology Publications and Royal Botanical Gardens, Kew, London, 337P.

2. Adebisi, A.A. (2004). A case study of Garcinia kola nut production-to consumption system in J4 area of Omo forest reserve, South-west Nigeria. - In: Sunderland, T. and Ndoye, O. (eds): Forest Products, Livelihood and Conservation (Case study of Non-Timber Forest Product Systems), Volume 2 Africa: 115-132.

3. Adepoju, A.A. \& Salau, A.S. (2007). Economic Valuation of NonTimber Forest Products. MPRA Paper No. 2689. Online at http:// mpra.ub.uni-muenchen.de/ 2689/. Accessed 08/08/2007.

4. Agali, AB. (2009). Diversité, structure et perception locales des espèces ligneuses fourragères dans le terroir de Torodi, Ouest Niger. Mémoire de DEA en Biologie et Ecologie Végétales. Université de Ouagadougou, Burkina Faso, 48p.

5. Aiyelaagbe, I.O., Labode Popoola, Adeola, A.O., Obisesan, K.O., \& Ladipo, D.O. (1996). Garcinia kola: its prevalence, farmer valuation, and strategies for its conservation in the rainforest of south eastern Nigeria. Paper contributed to the workshop on the Rainforest of Southeastern Nigeria and Southwestern Cameroon. 21-23 October, Cross River National Park, Obudu Ranch, Nigeria.Alexandre, D.Y. (1989). Dynamique de la régénération naturelle en forêt dense de Côted'Ivoire, Etudes et thèses, Paris ORSTOM, 102 P.

6. Calderon-Aguilera, L.E., Rivera-Monroy, V. H., Porter-Bolland, L., Martinez-Yrizar, A., Ladah, L. B., Martinez-Ramos, M., Alcocer, J., 
Santiago-Pérez, A. L., Hernandez-Arana, H. A., Reyes-Gomez, V.M., Pérez-Salicrup, D. R.,

7. Diaz-Nunez, V., Sosa-Ramirez, J., Herrera-Silveira, J., \& Burquez, A. (2012). Anassessment of natural and human disturbance effects on Mexican ecosystems: current trends and research gaps. Biodiversity and Conservation. 21:589-617.

8. Chazdon, R. L., Finegan, B., Capers, R.S., Salgado-Negret, B., Casanoves, F., Boukili, V., \& Norden, N. (2010). Composition and Dynamics of Functional Groups of Trees during tropical forest Succession in Northeastern Costa Rica. Biotropica 42(1): 31-40.

9. Condit, R. (1995). Research in large, long term tropical forest plots. Ecology and evolution 10 (1): 18-22.

10. De Wasseige, C., de Marcken, P., Bayol, N., Hiol Hiol, F., Mayaux, P., Desclée, B., Nasi, R., Billand, A., Defourny, P., \& Eba'a Atyi, R. (2012). Les Forêts du Bassin du Congo - État des Forêts 2010. Luxembourg.

11. Dijk, J.F.W. \& Wiersum, K.F. (1999). NTFP resource management as an option for multiple-use forest management in South Cameroon. In: Ros-Tonen M.A.F. (ed). NTFP research in the Tropenbos Programme: results and perpectives, 115-122.

12. Djuikouo, M.N.K., Doucet, J.L., Nguembou, C.K., Lewis, S., \& Sonke, B. (2010). Diversity and aboveground biomass in three tropical forest types in the Dja Biosphere Reserve, Cameroon. African Journal of Ecology. 48:1053-1063.

13. Dupuy, J.M. \& Chazdon, R.L. (2006). Effects of vegetation cover on seedling and sapling dynamics in secondary tropical wet forests in Costa Rica. Journal of TropicalEcology. 22, 65-76.

14. Eba'a Atyi, R., Devers, D., Wasseige, C., \& Maisel, F. (2008). État des forêts d'Afrique centrale : synthèse sous-régionale. In : Hall J. et al., eds. Les forêts du Bassin du Congo : état des forêts. Luxembourg: Office des Publications de l'Union européenne, 17-44.

15. Egbe, E.A., Tabot, P.T., \& Fonge, B.A. (2012). EthnobotanyandPrioritizationof Some Selected Tree Species in Southwestern Cameroon.Ethnobotany Research \& Applications. Volume 10:235-246.

16. Gemerden, BS. (2004). Disturbance, diversity and distributions in Central African rain forest.Ph.D thesis. Department of Environmental Sciences. The Netherlands: Wageningen University:74-81pp.

17. Gentry, A.H. (1988). Changes in plant community diversity and floristic composition on geographic and environmental gradients. Annals of the Missouri Botanical Garden. Vol.75, 1-34. 
18. Guedje, M.N. (2002). La gestion des populations d'arbres comme outil pour une exploitation durable des produits forestiers non ligneux : l'exemple de Garcinia lucida (Sud Cameroun). Thèse de doctorat : Univ. Libre de Bruxelles (Belgique). 226p.

19. Guedje, M.N., Nkongmeneck, B.A., \& Lejoly, J. (2002). Composition floristique et structure des formations à Garcinia lucida dans la region de Bipindi, Akom II (Sud Cameroun). Acta Botanica Gallica, 149 (2), 157-178.

20. Gyimah, A. (2000). Effect of pretreatment methods on germination of Garcinia kola Heckel seeds. Ghana Journal of Forestry. Vol. 9:39-44.

21. Hall, J.B. \& Swaine, M.D. (1981). Distribution and Ecology of Vascular Plants in a Tropical rian forest : Forest vegetation in Ghana.383p.

22. Hitimana, J., Kiyiapi, J.L., \& Njunge, J.T. (2004). Forest structure characteristics in disturbed and undisturbed sites of Mt. Elgon Moist Lower Montane Forest, western Kenya. Forest Ecology and Management 194, 269-291.

23. Illoldi-Rangel, P. \& Escalante, T. (2008). De los modelos de nicho ecológico a las áreas de distribución geográfica. Biogeografía 3:7-12.

24. INS (Institut National de Statistique) (2010). Rapport régional de progrès des objectifs du Millénaire pour le développement. Région de l'Est Cameroun. www.statistics-cameroon.org.32p.

25. IUCN (2004). IUCN Red List of Threatened Species, 217p.

26. Kémeuzé, V.A., Solefack, M.C.M., Nkongmeneck, B., Decocq, G., Jiofack, T., \& Johnson, M. (2009).Variation altudinale de la distribution des plantes à activité insecticide dans la forêt communautaire de Kilium- Ijium : cas de Clausena anisata. Bois et forêt des tropiques, $\mathrm{N}^{\circ} 299$ : 75-76.

27. Kessler, M., Kessler, P.J.A., Gradstein, S.R., Bach, K., Schmull, M., \& Pitopang, R. (2005). Tree diversity in primary forest and different land use systems in Central Sulawesi, Indonesia. Biodiversity and Conservation. Volume 14, 547-560.

28. Laurance, W.F., Alonso, A., Lee M., \& Campbell, P. (2006). Challenges for forest conservation in Gabon, Central Africa. Futures 38: 454-470.

29. Letouzey, R. (1985). Notice de la carte phytogéographique du Cameroun au 1:500 000. 2. Régionafromontagnarde et étage submontagnard. Institut de la Carte Internationale de la VégétationIRA (Herbier National), Yaoundé, Cameroun. 61 p.

30. Linder, H.P. (2001). Plant diversity and endemism in sub-Saharan tropical Africa. Journal of Biogeography. 28: 169-182. 
31. Liwen Zhang, Nurvianto, S., \& Harrison, R. (2010). Factors affecting the distribution and abundance of Asplenium nidus L. in a tropical lowland rain forest in Peninsular Malaysia.Biotropica 42(4):464-469.

32. Loubelo, E. (2012). Impact des Produits Forestiers Non Ligneux (Pfnl) sur L'Economie des Ménages et la Sécurité alimentaire : Cas de la République du Congo. Thèse de Doctorat: Ecole Doctorale « Sciences Humaines et Sociales » de l'Univ. Rennes 2. 260 p.

33. Lourmas, M. (2003). Diversité génétique et aménagement : utilité d'une modélisation intégrée. Bois et Forêts des Tropiques 276(2): 85 -87 .

34. Luna Vega, I. \& Magallon, S. (2010). Phylogenic composition of angiosperm diversity in the GRoud forests of Mexico. Biotropica 42 (4): 444-454.

35. Maître, H.F. (1986). Dynamique et production des peuplements naturels de forêt dense humide en Afrique. Bois et forêt des Tropiques 213, 3-12.

36. Martínez-Meyer, E. (2015). Climate change and biodiversity: some considerations in forecasting shifts in species' potential distributions. Global Biodiversity Information Facility.2:42-55.

37. Momo, S.M.C., Kamga, Y.B., Tiokeng, B., Nguetsop, V.F., Avana, M.L., \& Kemeuze, V. (2017). Diversité Floristique Et Variation Altitudinale De La Structure Des Formations A Gnidia Glauca (Fresen) Gilg. Dans Les Forêts Communautaires De Kilum-Ijim (Nord-Ouest Cameroun). European Scientific Journal.vol 13(6).21p.doi: 10.19044/esj.2017.v13n6p17

38. Naing, Z.H., Nobuya, M., \& Shigejiro, Y. (2011). Tree species composition and diversity at different levels of disturbance in Popa Mountain park, Myanmar. Biotropica 43(5): 597-603.

39. Ndoye, O. (1995). The market of non-timber forest products in the humid forest zone of Cameroon and its borders.Structure, conduct, performance and policy implications.CIFOR, Yaoundé, Cameroon. $86 \mathrm{p}$.

40. Ndoye, O., Ruiz-Perez, M., \& Eyebe, A. (1999). Non-Wood Forest Product Markets and Potential Degradation of the Forest Resource in Central Africa: the Role of research in providing a balance between welfare improvement and forest conservation. In Sunderland T.C.H., Clark L.E. \& Vantomme P. (eds.) Non-Wood Forest Products of Central Africa - Current Research Issues And Prospects For Conservation And Development: 183-206. Rome, FAO.

41. Ngueguim, J. R. (2013). Productivité et diversité floristique des ligneux dans les forêts denses d'Afrique tropicale du Cameroun : site 
de Mangombe, Bidou et Campo. Thèse de doctorat. Museum national d'histoire naturelle. Ecologie forestière. $213 \mathrm{p}$.

42. Onana, J. \& Devineau, J.-L. (2002). Afzelia africana Smith ex persoon dans le Nord Cameroun. Etat actuel des peuplements et utilisation pastorale. Revue d'Elevage et de Médecines Vétérinaire des PaysTropicaux 55(1) :39-45.

43. Ouédraogo, A.S. \& Boffa, J.M. (1999).Vers une approche régionale des ressources génétiques forestières en Afrique sub-saharienne. Actes $\mathrm{du}$ premier atelier régional de formation sur la conservation et l'utilisation durable des ressources génétiques forestières en Afrique de l'Ouest, Afrique Centrale et Madagascar, Centre National de Semences Forestières (CNSF), Ouagadougou, Burkina Faso du 16-27 Mars 1998. IPGRI-Rome-Italy, 299 p.

44. Ouédraogo, I. (2008). Diversité des espèces ligneuses utiles de la région du Nord du Burkinia Faso: état des peuplements de cinq espèces d'importance socio-économique. Mémoire d'ingénieur en Eaux et Forêts. Univ. de Bobo-Dioulasso, Burkinia Faso, 68p.

45. Parmentier, I., Harrigan, R.J., Buermann, W., Mitchard, E.T.A., Saatchi, S., Malhi, Y., Bongers, F., Hawthorne, W.D., Leal, M.E., Lewis, S.L., Nusbaumer, L., Sheil, D., Sosef, M.S.M., Affum-Baffoe, K., Bakayoko, A., Chuyong, G.B., Chatelain, C., Comiskey, J.A., Dauby, G., Doucet, J.L., Fauset, S., Gautier, L., Gillet, J.F., Kenfack, D., Kouame, F.N., Kouassi, E.K., Kouka, L.A., Parren, M.P.E., Peh, K.S.H., Reitsma, J.M., Senterre, B., Sonke, B., Sunderland, T.C.H., Swaine, M.D., Tchouto, M.G.P., Thomas, D., Van Valkenburg, J.L.C.H., \& Hardy, O.J. (2011). Predicting alpha diversity of African rain forests: models based on climate and satellite-derived data do not perform better than a purely spatial model. Journal of Biogeography.doi:10.1111/j.1365-2699.2010.02467.x

46. Plana, V. (2004). Mechanisms and tempo of evolution in the African Guineo-Congolian rainforest. Philos Trans R Soc Lond B Biol Sci 359: 1585-1594. doi: 10.1098/rstb.2004.1535

47. Puig, H. (2001). La Forêt Tropicale Humide. Berlin, Paris, 448p.

48. Ukizintambara, T., Lee, W., Kate, A., \& Thebaud, C. (2007). Gallery forests versus bosquets: conservation of natural fragments at Lope' National Park in central Gabon. African Journal of Ecology. 45, 476482.

49. Sacandé, M., Prichard, H.W., \& Dulloo, E.M. (2004).Seed Science and technology needs of SAFORGEN trees for conservation and sustainable use.Plant Genetic Resources. Newsletter 139: 53-58.

50. Senterre, B. (2005). Recherches méthodologiques pour la typologie de la végétation et la phytogéographie des forêts denses d'Afrique 
tropicale. Thèse de Doctorat, Univ. Libre de Bruxelles, Belgique, 345 p.

51. Steentoft, M. (1988). Flowering plants in West Africa.Cambridge University Press, $344 \mathrm{p}$

52. Thapa, S. \& Chapman, D.S. (2010). Impacts of resource extraction on forest structure and diversity in Bardia National Park, Nepal.Forest Ecology and Management. 259: 641-649.

53. Vivien J. \& Faure, J.J. (1996). Fruitier Sauvage d'Afrique: espèces du Cameroun. CTA et Ministère Français de la Coopération, 416 p.

54. White, F.(1986). La végétation de l'Afrique Mémoire accompagnant la carte de végétation de l'Afrique Unesco/AETFAT/UNSO. Orstom Unesco 16: 391.

55. Wong, J.L.G. (1997). The State of Ghana's Forests 1986-1997: NonTimber Forest Products.Fountain Renewable Resources Ltd., UK, 29 p.

56. Xiao-Tao Lü \& Jian-Wei Tang (2010). Structure and composition of the understory treelets in a non-dipterocarp forest of tropical Asia. Forest Ecology and Management 260:565-572.

57. Yemadje, P.L. (2011). Diversité floristique et biomasse des ligneux en forêt dense humide équatoriale: Cas des stations Forestières de Mangombé- Edéa et Bidou (Cameroun)-Thèse de Master. Univ. de Dschang-30p.

58. Zapfack, L., Engwald, S., Sonke, B., Achoundong, G., \& Birang à Madong (2002). The impact of land conversion on plant biodiversity in the forest zone of Cameroon. Biodiversity and Conservation 11: 2047-2061. 


\section{ANNEXES}

Liste des espèces rencontrées dans les formations à Garcinia kola dans les différents régions au cours de nos études classés suivant les critères morphologiques et importants pour assurer la régénération des espèces (Raunkiaer,1934 ; Dansereau et \& Lems, 1957 ; Doucet, 2003 ; Senterre, 2005 ; Meunier et al. 2015 ). TB= Type Biologique; TM= Type morphologique ; TD : Type de Diaspores ; MD : Mode de dissémination ; $\mathrm{A}=$ Arbre ; $\quad \mathrm{Ar}=\quad$ Arbuste ; $\mathrm{Nph}=\quad$ Nanophanérophytes ; $\mathrm{MgPh}=$ mégaphanérophytes ; $\mathrm{MsPh}=$ mésophanérophytes ; $\mathrm{McPh}=$ microphanérophytes.

\begin{tabular}{|c|c|c|c|c|c|}
\hline Familles & Espèces & TM & TB & TD & MD \\
\hline Fabaceae & Acacia sp & $\mathrm{Ar}$ & $\mathrm{MsPh}$ & & \\
\hline Huaceae & Afrostyrax kamerunensis Perkins \& Gilg, & A & $\mathrm{MsPh}$ & Sarco & Endozoo/Auto \\
\hline Huaceae & Afrostyrax lepidophyllus Mildbr., & A & $\mathrm{MsPh}$ & Sarco & Endozoo/Auto \\
\hline Caesalpiniaceae & Afzalia pachyloba Harms, & A & $\mathrm{MgPh}$ & Sarco & Endozoo \\
\hline Caesalpiniaceae & Afzelia bipindensisL. & A & $\mathrm{MgPh}$ & Sarco & Endozoo \\
\hline Mimosaceae & Albizia adianthifolia (Schum.) W.F.Wight & $\mathrm{Ar}$ & $\mathrm{MgPh}$ & Baro & auto \\
\hline Mimosaceae & Albizia ferruginea (Guill.\& Perr.)Benth. & A & $\mathrm{MgPh}$ & Baro & Auto \\
\hline Mimosaceae & Albizia gummifera (J.F.Gmel.) & A & Mph & Baro & Auto \\
\hline Mimosaceae & Albizia zygia (DC.) J. F. Macbr. & A & $\mathrm{MgPh}$ & Baro & Auto \\
\hline Euporbianceae & Alchornea cordifolia (Schum.\& Thonn.)Mull. & $\mathrm{Ar}$ & $\mathrm{McPh}$ & Ballo & Endozoo \\
\hline Clusiaceae & Allablackia floribunda Oliv. & $\mathrm{Ar}$ & $\mathrm{McPh}$ & Sarco & \\
\hline Clusiaceae & & $\mathrm{Ar}$ & $\mathrm{McPh}$ & Sarco & \\
\hline Sapindaceae & $\begin{array}{l}\text { Allablankia klainei } \text { Pierre ex A.Chev. } \\
\text { Allophylus bullatus }\end{array}$ & A & Msph & Sarco & Endozoo \\
\hline Apocynaceae & Alstonia boonei De Wild. & A & $\mathrm{MgPh}$ & Pogo & Ane \\
\hline Fabaceae & Amphimas ferrugeneus Pierre ex Pellegr. & A & $\mathrm{MgPh}$ & & Ane \\
\hline Fabaceae & Angylocalix sp & $\mathrm{Ar}$ & $\mathrm{MgPh}$ & Sarco & \\
\hline Annonaceae & annikia affinis (Exell) Versteegh \& Sosef, & A & $\mathrm{MgPh}$ & Sarco & Endozoo \\
\hline Annonaceae & Annonidium manni (Oliv.) Engl. \& Diel, & A & $\mathrm{MgPh}$ & Baro & Endozoo \\
\hline Caesalpiniaceae & Anthonotha macrophyllaP.Beauv, & A & $\mathrm{MsPh}$ & Bal & Auto \\
\hline Loganiaceae & Anthocleista cf. vogelii Planchon & $\mathrm{Ar}$ & $\mathrm{MgPh}$ & & \\
\hline Anacardiaceae & Antrocaryon klaineanum Pierre, & $\mathrm{A}$ & $\mathrm{MgPh}$ & Sarco & Endozoo \\
\hline
\end{tabular}




\begin{tabular}{|c|c|c|c|c|c|}
\hline Anacardiaceae & Antrocaryon micraster A.Chev. \& Guillaumin & A & $\mathrm{MgPh}$ & Sarco & Endozoo \\
\hline Sapotaceae & Baillonelala toxisperma Pierre & A & $\mathrm{MgPh}$ & Sarco & Endozoo \\
\hline Passifloraceae & Barteria fistulosa Mast., & A & Msph & Sarco & Endozoo \\
\hline Lauraceae & Beilschmiedia $\mathrm{sp}$ & A & Msph & Sarco & Endozoo \\
\hline Sapindaceae & Blighia welwitschii (Hiern) Radlk & A & $\mathrm{MgPh}$ & Sarco & \\
\hline Fabaceae & $\begin{array}{l}\text { Bobgunnia fistuloides(Harms) J.H. } \\
\text { Kirbr.\&Wiersema, }\end{array}$ & A & Msph & Sarco & sarco \\
\hline Euphorbiaceae & Bridelia ferrugeneaBenth. & A & Mcph & Sarco & Endozoo \\
\hline Euphorbiaceae & Bridelia speciosa & A & Mcph & Sarco & Endozoo \\
\hline Burseraceae & Canarium schweinfurthii Engl., & A & $\mathrm{MgPh}$ & Sarco & Endozoo \\
\hline Meliaceae & Carapa procera $\mathrm{DC}$ & $\mathrm{A}$ & Mph & Sarco & Endozoo \\
\hline Malvaceae & Ceiba pentandra (L.) G aertn. & A & $\mathrm{MgPh}$ & Sarco & Endozoo \\
\hline Ulmaceae & Celtis midbraedii Engl. & $\mathrm{A}$ & $\mathrm{MgPh}$ & Sarco & Endozoo \\
\hline Ulmaceae & Celtis philippensis Blanco & A & $\mathrm{MgPh}$ & Sarco & Endozoo \\
\hline Ulmaceae & Celtis sp & A & $\mathrm{MgPh}$ & Sarco & Endozoo \\
\hline Ulmaceae & Celtis tessmannii Rendle & A & $\mathrm{MgPh}$ & Sarco & Endozoo \\
\hline Ulmaceae & Celtis zenkeri Engl. & A & $\mathrm{MgPh}$ & Sarco & Endozoo \\
\hline Sapotaceae & Chrysophyllum africanum A.DC. & A & $\mathrm{MgPh}$ & Sarco & Endozoo \\
\hline Rutaceae & Citrus limon (L.) Burm.f.(cult.) & $\mathrm{Ar}$ & $\mathrm{McPh}$ & Sarco & Endozoo \\
\hline Rutaceae & Citrus reticulata Blanco & $\mathrm{Ar}$ & $\mathrm{McPh}$ & Sarco & Endozoo \\
\hline Rutaceae & Citrus grandis & $\mathrm{Ar}$ & $\mathrm{McPh}$ & Sarco & Endozoo \\
\hline Rutaceae & Citrus parasidisii Macf. (cult) & $\mathrm{Ar}$ & $\mathrm{McPh}$ & Sarco & Endozoo \\
\hline Rutaceae & Citrus sinensis (L.) Obs. (Cult.) & $\mathrm{Ar}$ & $\mathrm{McPh}$ & Sarco & Endozoo \\
\hline Myristicaceae & Coelocaryon preussiiWarb. & A & $\mathrm{MgPh}$ & Sarco & Endozoo \\
\hline Rubiaceae & Cofea robusta & $\mathrm{Ar}$ & Mph & Sarco & Endozoo \\
\hline Malvaceae & Cola accuminata (P.Beauv.) Schott \& Endl. & A & Msph & Sarco & Endozoo \\
\hline Malvaceae & Cola lateritia K.Schum. & A & Msph & Sarco & Endozoo \\
\hline Malvaceae & Cola nitida (Vent.) Schott \& Endl. & A & Msph & Sarco & Endozoo \\
\hline Malvaceae & Cola sp & A & Msph & Sarco & Endozoo \\
\hline Boraginaceae & Cordia africana Lam. & A & $\mathrm{MgPh}$ & & \\
\hline Rubiaceae & Corynanthe pachycerasK.Schum & A & $\mathrm{MgPh}$ & & \\
\hline Fabaceae & Cylicodiscus gabunensisHarms & A & $\mathrm{MgPh}$ & Sarco & Ane \\
\hline Burseraceae & Dacryodes edulis(G.Don) H. J. Lam & A & $\mathrm{MgPh}$ & Sarco & Endozoo \\
\hline Irvingiaceae & Desbordesia glaucescens (Engl.) Tiegh. & A & $\mathrm{MgPh}$ & Sarco & Ane \\
\hline Caesalpiniaceae & Detarium macrocarpum Harms & A & $\mathrm{MgPh}$ & Sarco & Endozoo \\
\hline Caesalpiniaceae & Dialium pachyphyllum Harms & A & $\mathrm{MgPh}$ & $\mathrm{Scl}$ & Endozoo \\
\hline Ebenaceae & Diospyros bipindensis GÜrke & A & $\mathrm{McPh}$ & Sar & Endozoo \\
\hline Ebenaceae & Diospyros crassifloraHiern & A & $\mathrm{McPh}$ & Sar & Endozoo \\
\hline
\end{tabular}




\begin{tabular}{|c|c|c|c|c|c|}
\hline Ebenaceae & Diospyros spp & $\mathrm{A}$ & $\mathrm{McPh}$ & Sar & Endozoo \\
\hline Fabaceae & Distemonanthus benthamianus Baill. & A & $\mathrm{MgPh}$ & Sarco & Ane \\
\hline Euphorbiaceae & Drypetes gossweileri S. Moore & $\mathrm{Ar}$ & $\mathrm{MsPh}$ & Sarco & \\
\hline Euphorbiaceae & Drypetes sp1 & $\mathrm{Ar}$ & $\mathrm{MsPh}$ & Sarco & \\
\hline Euphorbiaceae & Drypetes $\mathrm{sp} 2$ & $\mathrm{Ar}$ & $\mathrm{MsPh}$ & Sarco & \\
\hline Euphorbiaceae & DrypetesklaineiPierre ex Pax & $\mathrm{Ar}$ & $\mathrm{MsPh}$ & Sarco & \\
\hline Tiliaveae & Duboscia macrocarpaBocq. & A & $\mathrm{MgPh}$ & Sarco & \\
\hline Annonaceae & Enantia chlorantha Oliv. & A & Msph & Sarco & Ane \\
\hline Meliaceae & Entandrophragma candollei Harms & A & $\mathrm{MgPh}$ & Ballo & Ane \\
\hline Meliaceae & Entandrophragma cylindricum Sprague & A & $\mathrm{MgPh}$ & Ballo & Ane \\
\hline Meliaceae & Entandrophragma angolense (Welw.) C.DC. & A & $\mathrm{MgPh}$ & Ballo & Ane \\
\hline Meliaceae & $\begin{array}{l}\text { Entandrophrahma utile (Dawe \& Sprague) } \\
\text { Sprague }\end{array}$ & A & $\mathrm{MgPh}$ & Ballo & Ane \\
\hline Fabaceae & Erythrophleum ivorense A.Chev. & A & $\mathrm{MgPh}$ & Ballo & Endozoo \\
\hline Fabaceae & Erythrophleum suaveolens Guill \& Perr.) Brenan & A & $\mathrm{MgPh}$ & Ballo & Endozoo \\
\hline Moraceae & Ficus etrangula & $\mathrm{A}$ & Msph & Sarco & Endozoo \\
\hline Moraceae & Ficus exasperata Vahl. & A & Msph & Sarco & Endozoo \\
\hline Moraceae & Ficus mисоsa Welw. Ex Ficalho & A & Msph & Sarco & Endozoo \\
\hline Moraceae & Ficus sp & A & Msph & Sarco & Endozoo \\
\hline Moraceae & Ficus vageliana (Miq.) Miq. & A & Msph & Sarco & Endozoo \\
\hline Moraceae & Ficus vogelli & A & Msph & Sarco & Endozoo \\
\hline Apocynaceae & Funtumia africana (Benth.) Stapf & $\mathrm{A}$ & Msph & Pogo & Ane \\
\hline Apocynaceae & Funtumia elastica (Benth.) Stapf & A & Msph & Sarco & Ane \\
\hline Clusiaceae & Garcinia kola Heckel & A & $\mathrm{MgPh}$ & Sarco & Endozoo \\
\hline Clusiaceae & Garcinia sp1 & $\mathrm{A}$ & $\mathrm{MgPh}$ & Sarco & Endozoo \\
\hline Clusiaceae & Garciniasp2 & A & $\mathrm{MgPh}$ & Sarco & Endozoo \\
\hline Fabaceae & Gilbertiodendron dewevrei (De Wild.) J.Léonard & A & $\mathrm{MgPh}$ & & Auto \\
\hline Annonaceae & $\begin{array}{l}\text { Greenwayodendron suaveolens (Engl. \& Diels) } \\
\text { Verdc. }\end{array}$ & $\mathrm{Ar}$ & Msph & & Endozoo \\
\hline Meliaceae & Guarea cedrata (A.Chev.) Pellegr & $\mathrm{A}$ & Ind & Sarco & \\
\hline Meliaceae & Guarea thompsoniiSprague \& Hutch & A & Ind & Sarco & \\
\hline Hypéricaceae & Harungana madagascariensis Lam. Ex Poir, & A & $\mathrm{McPh}$ & Sarco & \\
\hline Olacaceae & Heisteria parvifolia $\mathrm{Sm}$. & A & $\mathrm{MgPh}$ & & \\
\hline Ulmaceae & Holoptelea grandis & A & $\mathrm{MgPh}$ & & \\
\hline Flacourtiaceae & Homalium le-testui Pellegr. & A & $\mathrm{McPh}$ & Sclé & \\
\hline Caesalpiniaceae & Hylodendron gabunense Taubert & A & $\mathrm{MgPh}$ & Ptéro & \\
\hline Hymenocardiaceae & Hymenocardia lyrata & $\mathrm{Ar}$ & Msph & & \\
\hline Hymenocardiaceae & Hymenocardia heudelotii & $\mathrm{Ar}$ & Msph & & \\
\hline
\end{tabular}




\begin{tabular}{|c|c|c|c|c|c|}
\hline Irvingiaceae & $\begin{array}{l}\text { Irvingia gabonensis (Aubrey-Lecomte ex O.Rorke) } \\
\text { Baill. }\end{array}$ & $\mathrm{A}$ & $\mathrm{MgPh}$ & Sarco & Endozoo \\
\hline Irvingiaceae & Irvingia grandifolia (Engl.) Engl. & A & $\mathrm{MgPh}$ & Sarco & Endozoo \\
\hline Meliaceae & Khaya ivorensis A.Chev & A & $\mathrm{MgPh}$ & Sarco & Endozoo \\
\hline Irvingiaceae & Klainedoxa gabonensis Pierre ex Engl. & A & $\mathrm{MgPh}$ & Sarco & Endozoo \\
\hline Anacardiaceae & Lannea welwitschii(Hiern) Engl & A & $\mathrm{MgPh}$ & & \\
\hline Méliaceae & $\begin{array}{l}\text { Leplaea cedrata (A. Chev.) E.J.M.Koenen \& J.J.de } \\
\text { Wilde }\end{array}$ & A & $\mathrm{MgPh}$ & Sarco & Endozoo \\
\hline Ochaceae & Lophira alata Banks ex C.F.Gaertn. & A & Msph & Pte & Ane \\
\hline Meliaceae & Lovoa trichilioides Harms & A & $\mathrm{MgPh}$ & Ptéro & Ane \\
\hline Euphorbiaceae & Macaranga assas Amougou & A & $\mathrm{McPh}$ & Sclé & Endozoo \\
\hline Euphorbiaceae & Macaranga barterie Müll.Arg. & A & $\mathrm{McPh}$ & Sclé & Endozoo \\
\hline Euphorbiaceae & Macaranga monodora & A & $\mathrm{McPh}$ & Sclé & Endozoo \\
\hline Euphorbiaceae & Macaranga staudtii $\mathrm{Pax}$ & A & $\mathrm{McPh}$ & Sclé & Endozoo \\
\hline Euphorbiaceae & Maesobotrya floribonda & A & $\mathrm{McPh}$ & Sclé & \\
\hline Euphorbiaceae & Maesobotryasp. & $\mathrm{Ar}$ & Msph & Sclé & \\
\hline Rhamnaceae & Maesopsis eminnii & A & $\mathrm{MgPh}$ & Sarco & \\
\hline Calophyllaceae. & Mammea africana Sabine & A & $\mathrm{MgPh}$ & Sarco & Endozoo \\
\hline Anacardiaceae & Mangifera indica $\mathrm{L}$. & A & $\mathrm{MsPh}$ & & \\
\hline Malvaceae & Mansonia altisima & A & $\mathrm{MgPh}$ & & \\
\hline Euphorbiaceae & Margaritaria discoidea (Baill.) Webster & A & Msph & Sarco & Endozoo \\
\hline Bignoniaceae & Markhamia lutea & A & $\mathrm{MsPh}$ & Ptéro & \\
\hline Bignoniaceae & Markhamia thomindosa & A & $\mathrm{MsPh}$ & Ptéro & \\
\hline Rubiaceae & Massularia acuminata(D.Don) Bull ex Hoyle & & & Sarco & \\
\hline Pandaceae & Microberlinia bisulcata A. Chev & A & $\mathrm{MgPh}$ & Ballo & \\
\hline Pandaceae & Microdesmis puberula & $\mathrm{Ar}$ & Msph & & \\
\hline Moraceae & Milicia excelsa(Welw.) C.C.Berg & A & $\mathrm{MgPh}$ & Sarco & Endozoo \\
\hline Papillionnaceae & Millettia laurentiiDe Wild & A & $\mathrm{MgPh}$ & Ballo & Auto \\
\hline Moraceae & Monodora miristica & A & $\mathrm{MgPh}$ & Ballo & \\
\hline Moraceae & Musanga cecropioides $\mathrm{R} . \mathrm{Br}$. & A & $\mathrm{McPh}$ & Sarco & Endozoo \\
\hline Urticaceae. & Myrianthus arboreus P. Beauv. & A & $\mathrm{MsPh}$ & Sarco & Endozoo \\
\hline Rubiaceae & Nauclea diderrichii(De Wild.\& T. Durand) Merr. & A & $\mathrm{MgPh}$ & Sarco & Endozoo \\
\hline Sterculiaceae & $\begin{array}{l}\text { Nesogorgonia papoverifera (A.Chev.) Capuron ex } \\
\text { N.Hallé }\end{array}$ & A & $\mathrm{McPh}$ & Ptéro & Ane \\
\hline Rhamnaceae & Octocosmus africanus & & & & \\
\hline Scytopetalaceae & Oubanguia africana Baillon & A & $\mathrm{MsPh}$ & Sarco & \\
\hline Annonaceae & \multicolumn{3}{|l|}{ Pachypodanthium staudtii(Engl. et Diels) Engl. et Diels } & Sarco & \\
\hline Pandaceae & Panda oleosa Pierre & A & $\mathrm{MgPh}$ & Sarco & Endozoo \\
\hline Sapindaceae & Pankovia lorenthii (De Wild.) Gilg ex De Wild & A & $\mathrm{MgPh}$ & Sarco & \\
\hline Fabaceae & Paraberlinia bifoliata ellegrin ????? & $\mathrm{Ar}$ & Msph & Ballo & \\
\hline
\end{tabular}




\begin{tabular}{|c|c|c|c|c|c|}
\hline Chrysobalanaceae & Parinari excelsa Sabine & $\mathrm{A}$ & $\mathrm{MgPh}$ & Sarco & Endozoo \\
\hline Sapindaceae & Pausinystalia macroceras (K. Schum.) Pierre & A & $\mathrm{MgPh}$ & Ptéro & Ane \\
\hline Fabaceae & Pentaclethra macrophylla Benth. & $\mathrm{A}$ & $\mathrm{MgPh}$ & Ballo & Auto \\
\hline Clusiaceae & Pentadesma butyraceaSabine & A & $\mathrm{MgPh}$ & Sarco & \\
\hline Lauraceae & Persea americana Mill. & A & Msph & Sarco & auto \\
\hline Lecythidaceae & Petersianthus macrocarpus (Beauv.) Liben & A & $\mathrm{MgPh}$ & Ptéro & Ane \\
\hline Apocynaceae & Picralima nitida (Stapf) Th. \& H.Durand & $\mathrm{Ar}$ & Msph & Sarco & Endozoo \\
\hline Mimosaceae & Piptadeniastrum africanum (Hook. F.) Brenan, & A & Msph & Ptéro & Ane \\
\hline Euphorbiaceae & Plagiostyles africana (Müll.Arg.) Prain & & & & \\
\hline Anacardiaceae & Pseudospondias microcarpa (A.Rich.) Engl. & A & Msph & Sarco & Endozoo \\
\hline Rubiaceae & Psychotria peduncularis (Salisb.) Steyerm. & Ar & $\mathrm{Nph}$ & Sarco & Endozoo \\
\hline Combretaceae & Pteleopsis hylodendronMildbr. & A & $\mathrm{MgPh}$ & Ptéro & ane \\
\hline Fabaceae & Pterocarpus soyauxii Taub. & A & $\mathrm{MgPh}$ & Ptéro & ane \\
\hline Malvaceae & Pterygota bequaertii De Wild., & A & Msph & Ptéro & ane \\
\hline Myristicaceae & Pycnanthus angolensis (Welw.) Warb. & A & $\mathrm{MgPh}$ & Sarco & Endozoo \\
\hline Euphorbiaceae & $\begin{array}{l}\text { Ricinodendron heudelottii (Baill.) Pierre ex } \\
\text { Heckel }\end{array}$ & A & $\mathrm{MgPh}$ & Sarco & Endozoo \\
\hline Euphorbiaceae & Rinodendron gabonensis & A & $\mathrm{MgPh}$ & Sarco & Endozoo \\
\hline Violaceae & Rinorea grandifolia & A & $\mathrm{MsPh}$ & Sclé & \\
\hline Rubiaceae & Rothmannia lujae & AR & $\mathrm{MsPh}$ & Sarco & \\
\hline Apocynaceae & Rauvolfia macrophylla & A & $\mathrm{MgPh}$ & & \\
\hline Apocynaceae & Rauvolfia vomitoria Afzel & $\mathrm{Ar}$ & Msph & Sarco & Endozoo \\
\hline Burseraceae & Santiria trimeria (Oliv.) Aubré. & A & Msph & Sarco & Endozoo \\
\hline Rubiaceae & Sarcocephalus pobeguinii Pobeg. & A & $\mathrm{MgPh}$ & Sarco & Endozoo \\
\hline Myristicaceae & Scyphocephalium mannii (Benth.) Warb. & A & $\mathrm{MgPh}$ & Sarco & Endozoo \\
\hline Anacardiaceae & Sorindeia grandifoliaEngl. & A & Msph & Sarco & \\
\hline Bignoniaceae & Spathodea campanulata P.Beauv. & A & Msph & Ptero & ane \\
\hline Myristicaceae & Staudtia kamerouniensis & A & $\mathrm{MgPh}$ & Sarco & Endozoo \\
\hline Malvaceae & Sterculia rhinopelatala & $\mathrm{Ar}$ & $\mathrm{MgPh}$ & Sarco & Endozoo \\
\hline Malvaceae & Sterculia tragacantha Lindl., & $\mathrm{Ar}$ & $\mathrm{MgPh}$ & Sarco & Endozoo \\
\hline Olacaceae & Strombosia grandifolia Hook.f., & A & Msph & Sarco & \\
\hline Olacaceae & Strombosia pustulata & A & Msph & Sarco & \\
\hline Strombosiaceae & Strombosiopsis tetandra & A & Msph & Sarco & \\
\hline Myrtaceae & Syzygium $\mathrm{sp}$ & A & Mph & & \\
\hline Apocynaceae & Tabernaemontana crassaBenth. & $\mathrm{A}$ & Msph & Sarco & \\
\hline Combretaceae & Terminalia superba Engl. Et Diels. & A & $\mathrm{MgPh}$ & Ptero & ane \\
\hline Fabaceae & Tessmania africana Harms & A & $\mathrm{MgPh}$ & Ballo & Ind \\
\hline Fabaceae & Tessmania annomala (Micheli) Harms & A & $\mathrm{MgPh}$ & Ballo & Ind \\
\hline
\end{tabular}




\begin{tabular}{|c|c|c|c|c|c|}
\hline Apocynaceae & Tetorchidium $s p$ & $\mathrm{Ar}$ & Msph & & \\
\hline Caesalpiniaceae & Tetraberlinia bifoliolata (Harms) Hauman & A & $\mathrm{MgPh}$ & Ballo & \\
\hline Fabaceae & $\begin{array}{l}\text { Tetrapleura tetraptera (Schumach. \& Thonn.) } \\
\text { Taub. }\end{array}$ & A & $\mathrm{MgPh}$ & & Endozoo \\
\hline malvaceae & Theobroma cacao $\mathrm{L}$. & $\mathrm{Ar}$ & $\mathrm{McPh}$ & & \\
\hline Strombosiaceae & Treculia africanaDecne, & A & Msph & Sarco & Endozoo \\
\hline Strombosiaceae & Treculia tragacantha & A & Msph & Sarco & Endozoo \\
\hline Ulmaceae & Trema orientalis (L.) Blume & $\mathrm{Ar}$ & Msph & Sarco & Endozoo \\
\hline Meliaceae & Trichilia monodelpha (Thonn.)J.J.de Wilde & $\mathrm{A}$ & Msph & Sarco & Endozoo \\
\hline Meliaceae & Trichilia monodora & A & Msph & Sarco & Endozoo \\
\hline Meliaceae & Trichilia rubescens Oliv. & A & Msph & Sarco & Endozoo \\
\hline Meliaceae & Trichilia tessmanni Harms & A & Msph & Sarco & Endozoo \\
\hline Anacardiaceae & Trichocypha arborea Engl. & $\mathrm{A}$ & Msph & Sarco & Endozoo \\
\hline Anacardiaceae & Trichoscypha accuminata Engl. & $\mathrm{A}$ & Msph & Sarco & Endozoo \\
\hline Sterculiaceae & Triplochiton scleroxylon K.Schum., & A & $\mathrm{MgPh}$ & Pte & Ane \\
\hline Euphorbiaceae & Uapaca guineensis Mûll. Arg., & A & Msph & Sarco & épizoo \\
\hline Euphorbiaceae & Uapaca heudelotii Baill., & A & Msph & Sarco & épizoo \\
\hline Euphorbiaceae & Uapaca mole Pax, & A & Msph & Sarco & épizoo \\
\hline Euphorbiaceae & Uapaca paludosa Aubrév. \& Léandri, & A & Msph & Sarco & épizoo \\
\hline Verbenaceae & Vitex doniana Hort. Brit. & A & Msph & Sarco & baro \\
\hline Verbenaceae & Vitex grandifolia & A & Msph & Sarco & baro \\
\hline Annonaceae & Xylopia aethiopica (Dunal) A.Rich., & A & Msph & Sarco & Endozoo \\
\hline Annonaceae & Xylopia kentazii Engl. \& Diels & A & Msph & Sarco & \\
\hline Annonaceae & Xylopia staudtii Engl. \& Diels & A & Msph & Sarco & \\
\hline Rutaceae & Zanthoxylum heitzii Aubrév. \& Pellegr. & A & $\mathrm{MgPh}$ & Pte & Endozoo \\
\hline Rutaceae & Zanthoxylum tessmanni Engl. & A & $\mathrm{MgPh}$ & Pte & Endozoo \\
\hline Rutaceae & Zanthozylum gilletii (De Wild.) P.G.Waterman & A & $\mathrm{MgPh}$ & Pte & Endozoo \\
\hline Apocynaceae & Voacanga africana(Scott-Elliot) Stapf & A & Msph & Sarco & Endozoo \\
\hline
\end{tabular}

